
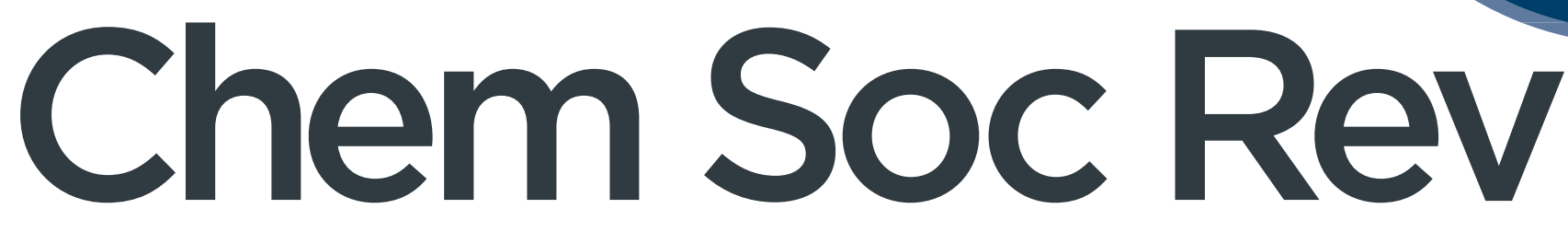

Volume 51

Number 1

7 January 2022

Pages 1-426

Chemical Society Reviews

rsc.li/chem-soc-rev

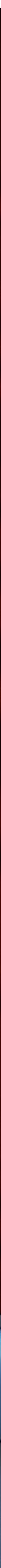

ISSN 0306-0012 
Check for updates

Cite this: Chem. Soc. Rev., 2022, 51,128

Received 10th August 2021

DOI: $10.1039 / d 1 c s 00686 j$

rsc.li/chem-soc-rev

\title{
Polymer nano-systems for the encapsulation and delivery of active biomacromolecular therapeutic agents
}

\author{
Marina Machtakova, ${ }^{a}$ Héloïse Thérien-Aubin (D) *ab and Katharina Landfester (D) *a
}

\begin{abstract}
Biomacromolecular therapeutic agents, particularly proteins, antigens, enzymes, and nucleic acids are emerging as powerful candidates for the treatment of various diseases and the development of the recent vaccine based on mRNA highlights the enormous potential of this class of drugs for future medical applications. However, biomacromolecular therapeutic agents present an enormous delivery challenge compared to traditional small molecules due to both a high molecular weight and a sensitive structure. Hence, the translation of their inherent pharmaceutical capacity into functional therapies is often hindered by the limited performance of conventional delivery vehicles. Polymer drug delivery systems are a modular solution able to address those issues. In this review, we discuss recent developments in the design of polymer delivery systems specifically tailored to the delivery challenges of biomacromolecular therapeutic agents. In the future, only in combination with a multifaceted and highly tunable delivery system, biomacromolecular therapeutic agents will realize their promising potential for the treatment of diseases and for the future of human health.
\end{abstract}

\section{Introduction}

The use of biological macromolecules, such as peptides, proteins, and nucleic acids as therapeutic agents is emerging as a powerful option for treating diseases like cancer, immunological and infectious diseases, or metabolic disorders. One of the cornerstones of the efficacy of this new class of drugs relies on their high specificity

\footnotetext{
${ }^{a}$ Max Planck Institute for Polymer Research, Ackermannweg 10, 55128 Mainz, Germany.E-mail: landfester@mpip-mainz.mpg.de, therien@mpip-mainz.mpg.de

${ }^{b}$ Department of Chemistry, Memorial University of Newfoundland, St. John's, NL, Canada.E-mail: htherienaubin@mun.ca
}

leading to highly decreased off-target effects. ${ }^{1-3}$ New treatments based on those biological macromolecules have benefited from extensive advances in molecular biology, which enabled the largescale production of such delicate biomolecules. This increased availability now offers an alternative to traditional small synthetic molecules, which often display both a low specificity and toxic side-effects in healthy tissues. ${ }^{4}$

Given the unique advantages of biomacromolecular drugs over small molecule therapeutic agents, their implementation in new treatment strategies represents one of the most promising fields of development in biochemical and pharmaceutical science.

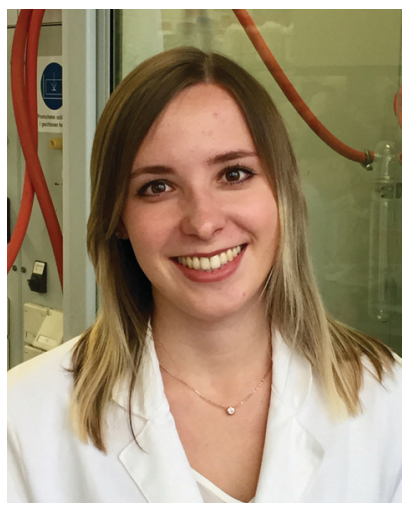

Marina Machtakova is currently a doctoral researcher at the Max Planck Institute for Polymer Research (MPIP). She obtained her BSc and MSc in chemistry from the Johannes-Gutenberg University in Germany. Her current research interest includes the synthesis of polymeric nanosystems for the delivery of active biomacromolecular therapeutic agents.

Marina Machtakova

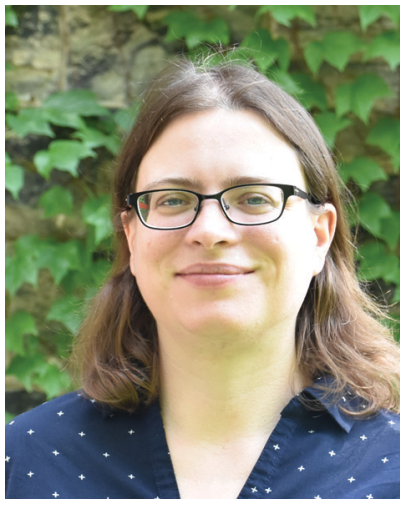

Héloïse Thérien-Aubin
Héloïse Thérien-Aubin is a professor in the Chemistry department at the Memorial University of Newfoundland, where her group study nanostructured polymer materials. She studied chemistry at the Universite de Montréal. After her PhD she was a postdoctoral fellow at Cornell University, and then, at the University of Toronto. From 2016 to 2021, she was a group leader at the MPIP. 
Despite the remarkable potential of biomacromolecular therapeutic agents (BTAs), only a few examples of such treatments have successfully translated to clinical practice. The successful examples include insulin for the treatment of diabetes, ${ }^{5}$ somatropin used in growth hormone therapy, ${ }^{6}$ or monoclonal antibodies approved for the treatment of cancer and other diseases. ${ }^{7}$ In fact, among the more than 20000 drugs currently approved by the FDA, less than $2 \%$ of them are BTAs. ${ }^{8}$ Although recent research developments have improved the efficiency of treatment based on the use of BTAs, successful clinical outcomes are rare. One of the crucial challenges currently encountered in many clinical trials is the inadequate and off-target delivery of these therapeutic agents. ${ }^{3,9,10}$ In particular, several studies attributed the failure of treatments based on the use of BTAs to their presumably poor delivery mechanism. ${ }^{11-13}$

Amid today's global health crisis caused by the severe acute respiratory syndrome coronavirus 2 (SARS-CoV-2), the need to design appropriate delivery systems for BTAs is more evident than ever. The new vaccines against COVID-19, like those developed by Pfizer/BioNTech and Moderna deliver the genetic sequence of the viral protein in the form of mRNA to the host cell. ${ }^{14}$ The mRNA induces the expression of the virus protein and leads to immunity against the original virus. However, using mRNA alone would lead to low efficacy, as mRNA is easily degraded by RNAses during circulation in the blood and cannot efficiently cross the cellular membrane due to its large size. ${ }^{15}$ If the mRNA vaccine formulation has been successful, it is in part due to the delivery vehicle, a lipid nanoparticle, a great achievement of nanotechnological engineering in and of itself, used as the delivery system. ${ }^{16}$ The encapsulation of the mRNA in the cationic lipid nanoparticles allows to protect the RNA from extracellular RNases and facilitates the uptake and endosomal release of the gene in the targeted cells. However, both the limited stability of the vaccine and the difficulties in scaling up the production are mainly associated with the ionizable lipid nanoparticles used as the delivery vehicle for the mRNA. ${ }^{17}$ It is clear that the scientific success of these vaccines providing

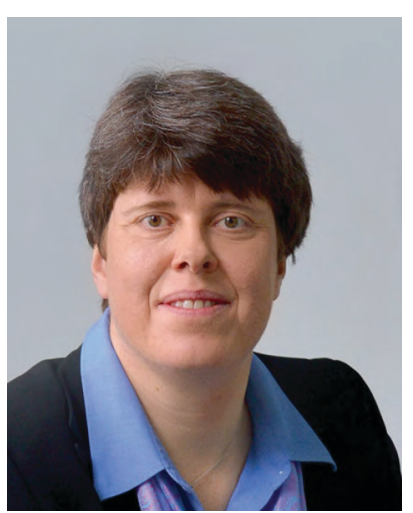

Katharina Landfester
Katharina Landfester received her doctoral degree in Physical Chemistry in 1995 after working with Prof. Spiess at the MPIP. After a postdoctoral stay at the Lehigh University, she worked at the MPI of Colloids and Interfaces in Golm leading the miniemulsion group. From 2003 to 2008 , she was a professor at the University of Ulm. She joined the Max Planck Society in 2008 as one of the directors of the MPIP. Her research focuses on creating functional colloids for new material and biomaterial applications. a way out of the global crisis results from a strong collaboration between bio- and nanotechnology. This success also clearly displays the potential and benefits of biomacromolecular therapeutics but also highlights the urgent need for the design of efficient, stable, and versatile delivery systems for those molecules. ${ }^{18}$ Polymer-based drug delivery systems are being used in an increasing manner. In the last few decades, the first examples of those systems were successfully translated from the labs to the clinical applications, for both the delivery of small molecules (e.g., Atridox ${ }^{\circledR}$ an antibiotic, Abraxane ${ }^{\circledR}$ used in the treatment of breast and lung cancer, Sublocade ${ }^{\circledR}$ used to treat opioid addiction) and biomacromolecular therapeutic agent (e.g., Oncaspar ${ }^{\circledR}$ to treat leukemia). ${ }^{19-22}$ Some of the challenges faced in the design of new polymer DDSs for biomacromolecular therapeutic agents are shared with the DDSs design for small molecules. The continuing success in translating polymer DDSs toward clinical applications hinges both the design of more effective and efficient DDSs and the standardization of the production processes. ${ }^{23}$

Here, we highlight the challenges for the delivery of biomacromolecular therapeutics and the solutions polymer nanocarriers may provide for their delivery. Although the prominent success of the mRNA delivery in COVID-19 vaccines was accomplished with lipid-based nanocarrier, multiple smart polymer nanostructures are emerging as effective alternatives. Such drug delivery systems (DDSs) provide unmatched diversity and control in their design. In the review, we define nanocarriers as structures used to deliver the drug to desired tissues, organs, cells, and subcellular organs and potentially released the drug in a determined manner. The usual purpose of nanocarriers is to improve the pharmacological properties of the therapeutic drugs and to overcome challenges such as limited stability, low bioavailability, poor biodistribution, or the lack of selectivity. Excellent reviews on the general use of polymer nanostructures in drug delivery can be found elsewhere, ${ }^{24-27}$ here, the focus is on their use in the delivery of biomacromolecular therapeutics such as proteins, enzymes, and oligonucleotides.

\section{Understanding the challenges in the delivery of active biomacromolecular therapeutic agents}

The unique performance of treatments based on BTAs arises from the combination of their composition and structure. However, the structure of large biological molecules such as proteins and nucleic acids is complex. Due to natural evolution, the sequence of the individual building blocks is hierarchically defined and adapted to the inherent biological function. Moreover, the functionalities of those molecules depend not only on the primary structure, their chemical composition but also on the secondary, tertiary, and quaternary structures, i.e., how the molecules are folded and organized in complex 3D assemblies. $^{28}$ Many non-covalent forces such as hydrogen bonds, van der Waals, electrostatic or hydrophobic interactions 
are present throughout the molecule and arrange the sequence of the building blocks in a specific three-dimensional folding. ${ }^{29}$ It is this unique structure that defines the activity and specificity of the BTAs. ${ }^{29,30}$

One of the crucial challenges in the delivery of BTAs is to preserve this inherent sensitive structure of the BTAs during the delivery process. ${ }^{31}$ In general, biological macromolecules are vulnerable to the loss of their structure due to the effect of physicochemical triggers such as temperature, $\mathrm{pH}$ value, and the ionic strength of the surrounding environment. Those conditions can disrupt the specific intra- and inter-molecular interactions based on labile forces responsible for their complex structures. These physicochemical cues can cause aggregation, deamidation, isomerization, hydrolysis, oxidation, and denaturation, resulting in the irreversible loss of biological activity. Moreover, the primary structure also needs to be protected since it can be subjected to enzymatic degradation in vivo. Various proteases and nucleases found in the biological media can degrade the molecules into smaller fragments. ${ }^{32,33}$ This is a critical factor that leads to short in vivo half-life times, ranging from a few minutes to a few hours, exhibited by some BTAs. ${ }^{34}$ This is one of the reasons why the use of BTAs as medications administered systemically, like in the case of insulin to treat diabetes, often requires frequent and constant dosing to maintain an appropriate in vivo concentration. ${ }^{35}$

Another key challenge is the delivery of the large biomacromolecules, used as therapeutic agents, across the cellular membrane to the cytoplasm. ${ }^{36}$ After billions of years of evolution, the cell membrane has evolved to precisely regulate the transport of molecules in and out of the cytosol to protect the intracellular environment from extracellular interference. ${ }^{37}$ The high molecular weight and the intrinsic hydrophilicity of many BTAs are associated with a general reduction in their permeability across biological barriers such as cell membranes. ${ }^{38}$ Cellular membranes can selectively and actively allow small molecules and ions to enter, while the lipid bilayer is impermeable to high-molecular-weight substances. ${ }^{39}$ The intrinsic lipophilic nature of biological membranes is another major obstacle in the permeation of hydrophilic biomacromolecules to the targeted intracellular site. Furthermore, in the case of nucleic acid-based drugs, the active molecules bear the same surface charge as the negatively charged cellular surface, making their transport across the membrane nearly impossible without the aid of external forces. ${ }^{40}$ As a result, only a marginal fraction of BTAs can successfully reach their intracellular target, which leads to insufficient therapeutic efficacy.

\section{Structures of polymer DDSs}

For the past 50 years, lipid nanoformulations have dominated the field of nanosystems for drug delivery. ${ }^{41}$ The approval of Doxil $^{\circledR}$ in 1995, a liposomal nanoformulation, is an important milestone in the development of nanomedicine. Originally developed as vehicles for the delivery of small molecules, liposomes and lipid formulations are now used to deliver a range of different payloads, including BTAs, as exemplified in the delivery of mRNA in COVID-19 vaccines. ${ }^{42,43}$ In this case, spherical lipid nanoparticles are made of ionizable lipids, which are able to complex the mRNA by electrostatic interactions. Due to the size and properties of the lipid nanoparticles, they are taken up by cells via endocytic pathways, and the cationic charge of the lipids at low $\mathrm{pH}$ values leads to the endosomal escape releasing the mRNA into the cell cytoplasm. The chemical composition of the lipid nanoparticles is very close to that of cell membranes, conferring to such DDSs excellent biocompatibility and biodegradability.

Although lipid nanoparticles and liposomes have been used to deliver both small molecules and BTAs, some limitations remain to their widespread applications. One reason is that those formulations are based on self-assembling lipids and can suffer from destabilization or dissociation in biological media. Such dynamic dissociation might lead to both side effects due to off-target activation and the loss of the valuable therapeutic agent due to its degradation outside of the dissociated nanostructure. Hence, this inherent instability is one of the Achilles' heels of lipid and liposomal delivery of BTAs. ${ }^{44,45}$ Another limitation in the design of liposomal delivery systems is the lack of structural diversity in the type of compatible lipid material used to generate the liposomes. The ideal drug delivery system might require the addition of smart functionalities such as stimuli-responsive structures for the precise and controlled release of the cargo or highly functional targeting moieties to overcome the hurdles toward efficient, specific, and targeted delivery.

Polymer nanostructures offer interesting alternatives. They provide a great versatility of building blocks and enable the incorporation of additional functionalities to the delivery systems. Furthermore, the organization of the polymer and the BTAs can adopt different structures leading to different DDSs architectures such as polymer-conjugates, polyplexes, layer-by-layer assemblies, nanogels, nanocapsules, and polymersomes (Fig. 1). The advances in the field have yielded a variety of structures with unique loading and functionalization, each displaying their own advantages for the successful delivery of BTAs (Table 1).

\subsection{Polymer-drug conjugates}

The conjugation of synthetic polymers with various BTAs is the simplest polymer DSS possible. Such polymer-drug conjugates are pharmacologically active constructs comprising one or more therapeutic agents covalently bound to a polymer chain. ${ }^{46}$ Since the first poly(ethylene glycol) (PEG)-protein conjugate, Adagen ${ }^{\circledR}$ (bovine pegademase), was approved by the FDA in $1990,{ }^{47}$ functional polymer bioconjugates have been widely explored and are continuously evolving. A commonly used model of a polymer-drug conjugate consists of a biocompatible watersoluble polymer backbone as the main delivery vehicle tethered to the biomacromolecular agent (Fig. 2a). In this case, the polymer chain increases the aqueous solubility of the drug and protects the cargoes from a rapid exclusion from the body. Several types of BTAs can be employed to design polymer conjugates, irrespective of their size, charge, or chemical structure. 


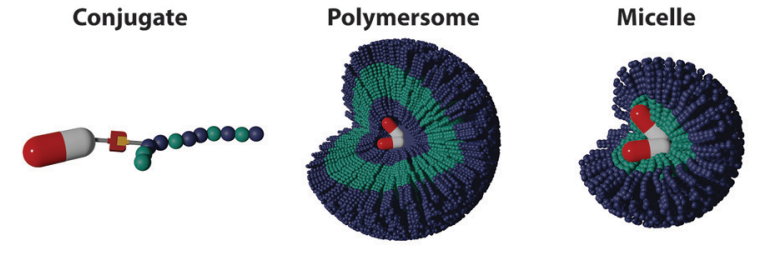

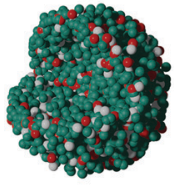

Polyplex

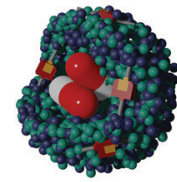

Nanoparticle

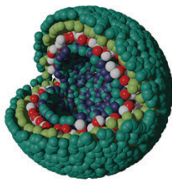

Layer-by-layer Assembly

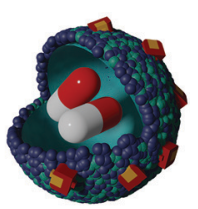

Nanocapsule
Biomacromolecular Therapeutic Agent

Polymer:

Fig. 1 Types of polymer DDSs for the encapsulation and delivery of biomacromolecular therapeutic agents.

Furthermore, one or several therapeutic molecules can be tethered to the same polymer backbone, and complementary moieties can also be attached to the polymer backbone, such as adjuvant drugs, fluorescent tags, PEG molecules, or targeting moieties.

Synthesis. Generally, there are two strategies to synthesize polymer conjugates. The "grafting to" method, the most common and straightforward methodology, where a preformed polymer is directly coupled to the target BTA. ${ }^{48}$ In this approach, amino and thiol groups present in the BTAs are frequently employed as conjugation sites. Amines can form amide bonds by reaction with activated esters such as an $\mathrm{N}$-succinimidyl activated ester or a pentafluorophenyl activated ester or a carboxylic acid activating coupling agent such as carbodiimide. ${ }^{49}$ Thiols can react with alkenes, maleimides, or vinyl sulphones to yield thioethers. ${ }^{50}$ For instance, a study analyzed the sitespecific grafting of polymers to the enzyme prolyl endopeptidase by reacting thiol groups introduced to the enzyme with maleimide-functionalized polymers. ${ }^{51}$ The authors focused specifically on the conjugation site and its influence on the enzymatic activity of the conjugates. Their findings clearly demonstrate that the conjugation of polymers at the catalytic site of the protein significantly decreases the enzymatic activity, while conjugations on other parts of the protein preserve a high degree of the activity (Fig. 2b). At the same time, the molecular weight of the polymer and its structure show a lower impact on the enzymatic activity when compared to the conjugation site. ${ }^{51}$

Alternatively, BTAs can be functionalized with nonendogenous amino acids or other appropriate external small molecules by using specific reactions. Those can be employed for the site-specific conjugation of polymers using bioorthogonal coupling chemistries. Many examples exploiting such a strategy exist. For instance, the unnatural amino acid $p$-azidophenylalanine was site-specifically incorporated into proteins. ${ }^{52}$ The azido end-group enabled a copper-medicated click-reaction with an alkyne functionalized PEG. In another example, a ketonecontaining amino acid, $p$-acetylphenylalanine was attached to a human growth hormone, and the conjugation of an aminooxy-functionalized PEG optimized the pharmacological properties of this therapeutic. ${ }^{53}$ Many advantages are associated with the "grafting to" approach. Firstly, the synthesis of the polymer may occur in a non-aqueous solution before the conjugation step.

Secondly, this separate synthesis process enables the design of well-defined polymer building blocks for preparing advanced materials with controlled structures, often including several reaction steps. Thirdly, a wide variety of (bioorthogonal) reactions is available to efficiently preserve the sensitive structures of the BTAs during the conjugation process. However, this approach also suffers from shortcomings, such as a potential low yield of the reaction between the polymer and the biomacromolecule. Here, two large molecules must react together, and the reaction can be limited due to steric hindrance leading to insufficient functionalization yields. ${ }^{50}$ Moreover, the resulting product can be challenging to purify from unreacted starting material and by-products. ${ }^{75}$

As a consequence, the "grafting from" method has emerged for the synthesis of polymer-drug conjugates. The development of the "grafting from" methodology has benefited from advances in controlled and living polymerization techniques, including the reversible addition-fragmentation transfer (RAFT) and the atom transfer radical polymerization (ATRP). ${ }^{76}$ Typically, the "grafting from" approach involves the conjugation of the initiator to a BTA. This leads to a formation of a macroinitiator for the following polymerization and leads to a controlled growth of the polymer conjugate. The overall process results in high reaction yield since only small molecules, first the initiator and then the monomers one at a time, are coupled to the reactive site. ${ }^{77}$ Still, this method may hamper BTAs. For example, the conjugation method employed to prepare proteinpolymer conjugates should not include reactive moieties undergoing side reactions with the amino acid functionalities of the protein. Additionally, the polymerization method should not lead to the denaturation of the BTAs, as induced by chemical modification or by inducing irreversible solubility changes. ${ }^{50}$

Controlled polymerizations, like ATRP and RAFT, have been extensively used to synthesize polymer with biomacromolecules since they combine a high degree of control over the resulting molecular weight and structure of the polymer chains and can be executed in aqueous media. ${ }^{50}$ Many BTAs have been functionalized in this way. For example, ATRP initiators were immobilized on proteins, which were used to initiate the growth of uniform poly(PEG-methacrylate) chains. ${ }^{78}$ In another example, the enzyme chymotrypsin was homogenously functionalized with nearly monodisperse polymer chains with a polydispersity index of 1.05. Furthermore, the protein-polymer conjugates synthesized by this method retained $50-86 \%$ of the original enzyme activity. ${ }^{79}$ Mild reaction conditions, using $\mathrm{Cu}(0)$-mediated ATRP, was used to form Candida antarctica lipase B (CALB) conjugated to different polymers of acrylamides and acrylates. ${ }^{55}$ The conjugates CALB-poly(hydroxyethyl acrylamide) (HEAA), CALB-poly( $N$-isopropylacrylamide) (NIPAM) 
Table 1 Overview of the different polymer nanostructures for the delivery of biomacromolecular therapeutic agents

\begin{tabular}{|c|c|c|c|c|}
\hline $\begin{array}{l}\text { Polymer } \\
\text { structure }\end{array}$ & Mode of formation & Example & Advantages & Limitations \\
\hline Conjugate & Covalent attachment & $\begin{array}{l}\text { PEGylation of proteins, } \\
5^{4} \\
\text { Stabilization of enzymes: }{ }^{55} \\
\text { PEGylated asparaginase (FDA/EMA } \\
\text { approved) }{ }^{56} \\
\text { Peginterferon } \alpha \text {-2b (FDA/EMA } \\
\text { approved) }{ }^{57,58}\end{array}$ & $\begin{array}{l}\text { - No leakage of the drug } \\
\text { - High drug/polymer ratio } \\
\text { - Low degree of potentially harm- } \\
\text { ful polymers } \\
\text { - Responsive conjugates enable } \\
\text { controlled release }\end{array}$ & $\begin{array}{l}\text { - Chemical modification of } \\
\text { biomolecule } \\
\text { - High risk of denaturation } \\
\text { - Limited steric protection }\end{array}$ \\
\hline LbL assembly & $\begin{array}{l}\text { Electrostatic } \\
\text { complexation of } \\
\text { charged molecules }\end{array}$ & $\begin{array}{l}\text { Co-delivery of antigens and } \\
\text { adjuvants }^{64} \\
\text { Co-delivery of gene and anticancer } \\
\text { drug }^{65}\end{array}$ & $\begin{array}{l}\text { - Absence of chemical modifica- } \\
\text { tion of the drug } \\
\text { - Increased cellular uptake due to } \\
\text { electrostatic interactions with the } \\
\text { cells }\end{array}$ & $\begin{array}{l}\text { - Limited to charged } \\
\text { biomolecules } \\
\text { - Electrostatic interactions } \\
\text { influenced by external } \\
\text { factors } \\
\text { - High risk of dissociation in } \\
\text { blood }\end{array}$ \\
\hline $\begin{array}{l}\text { Micelle and } \\
\text { polymersome }\end{array}$ & $\begin{array}{l}\text { Physical } \\
\text { encapsulation }\end{array}$ & $\begin{array}{l}\text { Controlled release of plasmidDNA } \\
\text { Targeted delivery of siRNA } \\
\text { mRNA delivery (animal study) } \\
\text { Polymersome delivery of the } \\
\text { SARS-CoV-2 spike protein } \\
\text { (preclinical trials) }^{69}\end{array}$ & $\begin{array}{l}\text { - Absence of chemical } \\
\text { modification of the drug } \\
\text { - Variety of morphologies and } \\
\text { architectures } \\
\text { - High protection }\end{array}$ & $\begin{array}{l}\text { - Limited encapsulation } \\
\text { efficiencies } \\
\bullet \text { High risk of dissociation in } \\
\text { blood }\end{array}$ \\
\hline
\end{tabular}

and CALB-poly(N-tert-butylacrylamide) (TBA) preserved the original biological activity of CALB, and the conjugates with poly(HEAA) and poly(NIPAM) even exhibited increased enzymatic activity by $\sim 1.5$ and 2 -fold. The lipolytic activity of CALBconjugate was measured by the rate of hydrolysis of nitrophenyl palmitate in heptane. The activity enhancement was ascribed ${ }^{80,81}$ to the increased solubility in organic media and increased affinity of the enzyme to the substrate in heptane after the formation of the polymer-conjugate. ${ }^{55}$

Examples of application. The versatility of such a conjugation approach enables the design of a highly multifunctional system with increased complexity tailored to the specific delivery challenge. ${ }^{82}$ In general, polymer-drug conjugates display high drug loading, sustained drug release, and enhanced stability without undesirable drug leaking. NKTR-214, a PEGylated interleukin-2 (IL-2) clinical candidate, is an example of the therapeutic potential of protein-polymer conjugates (Fig. 2c). ${ }^{83}$ The interleukin-2 protein is an activator and suppressor of the immune system and thus a key component for inducing a durable anti-tumor response in patients with advanced melanoma. However, due to its short half-life, it requires a high dose and frequent administration, which leads to an over-activation of the immune system and severe toxicity. NKTR-214, a multi-PEGylated IL-2 form, was synthesized to 
a

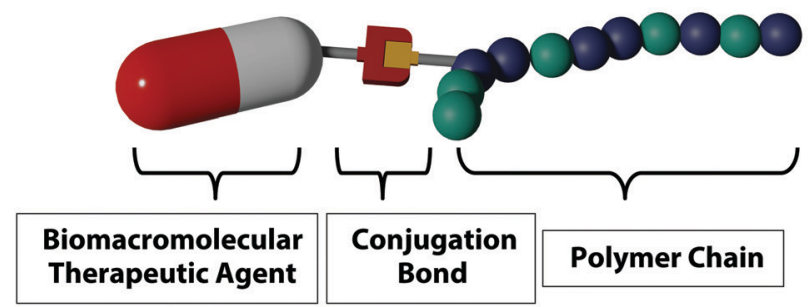

b
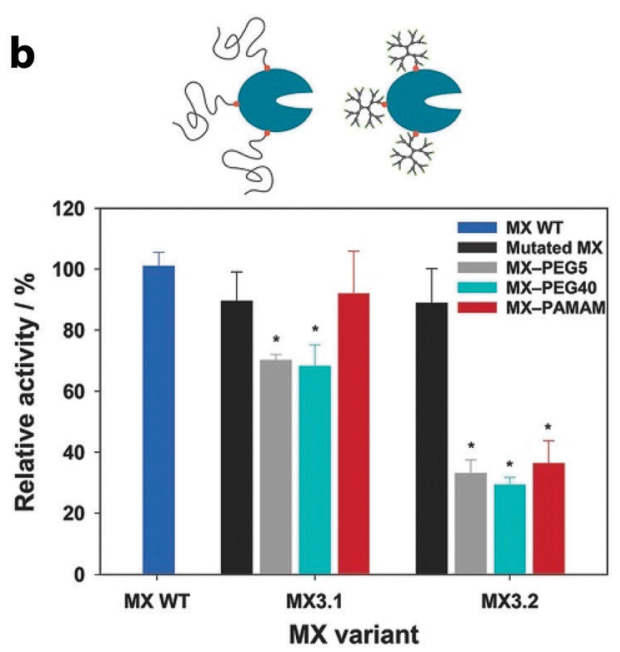

PEG
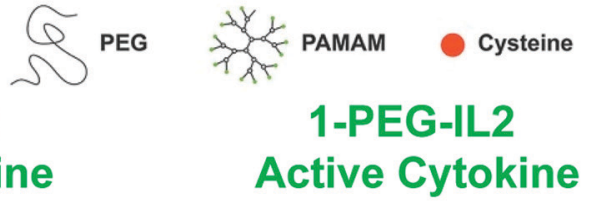
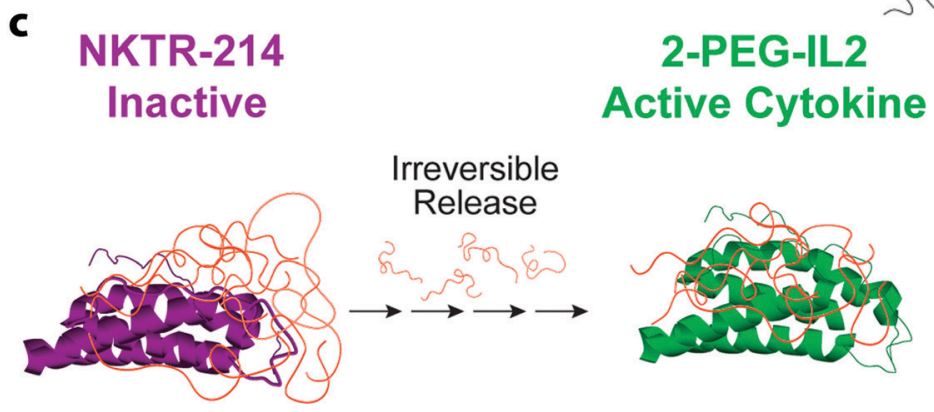

\section{Irreversible}
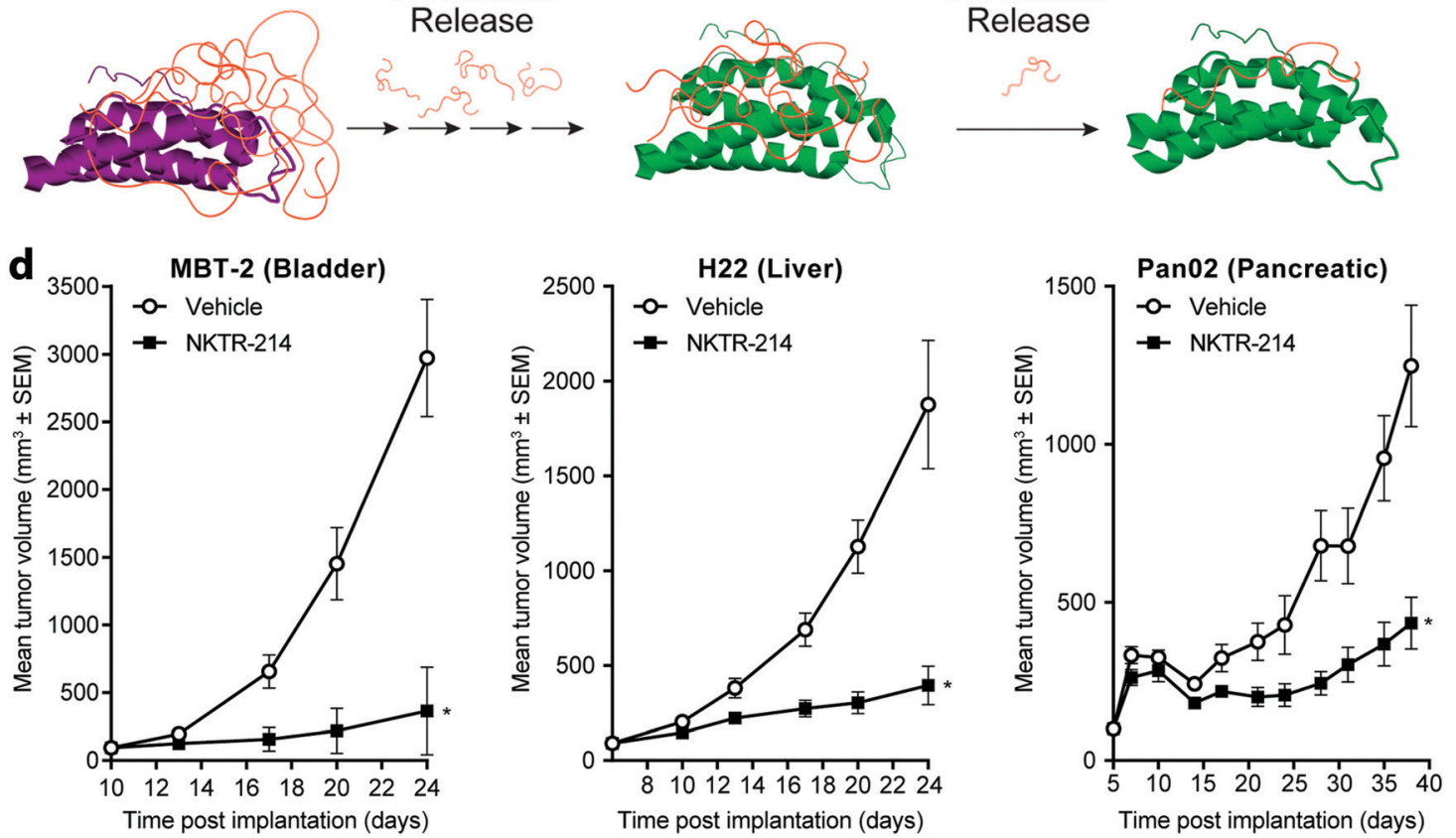

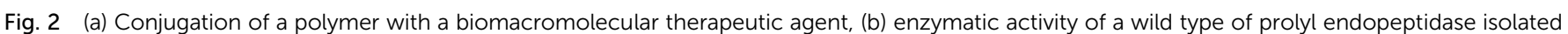

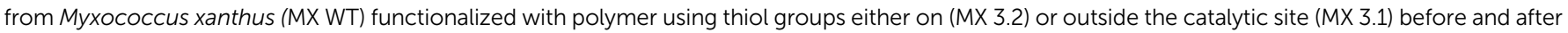

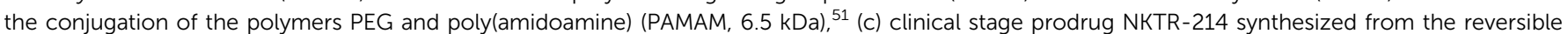

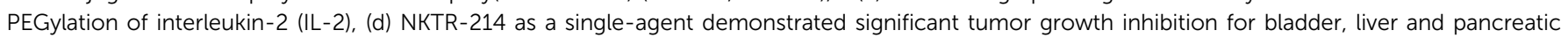
carcinoma. (b) Reprinted with permission from ref. 51 Copyright 2016 Wiley; (c and d) reprinted with permission from ref. 83 . Copyright 2017 PLOS.

harness the immune stimulatory benefits of the protein to increase the anti-tumor responses while minimizing undesired therapeutical side effects. ${ }^{84}$ In this PEG-functionalized form, the IL2 is inactive and a biological prodrug. When administered in vivo, the PEG chains are slowly cleaved from the IL-2, releasing and producing active IL2 conjugates bound by fewer PEG chains. The analysis of the tumor pharmacokinetics of
NKTR-214 and its active conjugated-IL2 forms were performed in mouse melanoma tumor tissues after the administration of a single dose of NKTR-214 or five doses of native Il-2 (aldesleukin). Tumor aldesleukin levels rapidly reached a maximal concentration and then rapidly declined. In contrast, NKTR-214 was detectable in tumors for up to 8 days after a single dose and achieved a 500-fold increased exposure relative to aldesleukin. ${ }^{84}$ 
In a further study, the in vivo antitumor efficacy of NKTR-214 was tested. Consistently, NKTR-214 as a single-agent demonstrated significant tumor growth inhibition across three tumor types tested: bladder (92\%), liver (79\%), and pancreatic $(65 \%)$ carcinoma (Fig. 2d). ${ }^{83}$

Several examples of polymer-conjugates with BTAs have already been approved by the US Food and Drug Administration and the European Medicines Agency. For instance, the PEGylated enzyme aspargase (Oncaspar ${ }^{\circledR}$ ) was approved to treat patients with acute lymphoblastic leukemia. The PEGconjugation of the enzyme increased its circulation time, which allowed for less frequent administration of the treatment to obtain the same response as the unmodified enzyme. ${ }^{56}$ Another formulation of the recombinant interferon alfa- $2 b$ protein with PEG was approved to treat patients with melanoma. ${ }^{57}$ The conjugation of the interferon to the PEG polymer protects the protein from degradation by enzymes and increases its half-life. The treatment with the PEGinterferon led to a sustained and clinically meaningful longer relapse-free survival time after the surgical tumor removal. ${ }^{58}$

Advantages and limitations. In polymer-drug conjugate, only a few polymer chains are attached to the drug; consequently, the ratio of drug to polymer is high, and the potential in vivo toxicity and the immuno-stimulatory effect that can arise from administering body-foreign material are limited in comparison to other delivery vehicles. ${ }^{85}$ Furthermore, especially when considering BTAs, the most promising benefit of developing polymer conjugates is the possibility to improve the stability of the drug. The stability of the BTA under diverse or extreme temperatures and $\mathrm{pH}$ ranges can be enhanced due to the steric shielding of the therapeutic agent by the polymer. ${ }^{86}$ This can also modulate the pharmacokinetic properties and increase the circulation time and cellular uptake of the drug. ${ }^{46}$ All of these benefits lead to an increased therapeutic effect observed for multiple drugs after their conjugation. ${ }^{87}$ However, during the conjugation process, the choice of the right chemistry is crucial in preserving the activity of the BTA. The direct covalent modification can alter the sensitive 3D structure of the biomacromolecular agent, even when extensive care is taken to preserve the specific folding of the drug during the conjugation process. Hence, this method always comes with a risk of denaturation and loss of functionality of the drug. Additionally, while BTAs encapsulated inside particles can be fully protected from degradation during their transport in vivo, polymerconjugates are still vulnerable to some degradation processes and generally show limited protection of the BTAs in comparison to other polymer delivery systems. ${ }^{46}$

\subsection{Coacervates and electrostatic polyplexes}

The formation of supramolecular structures between two polymers based on specific interactions such as electrostatic, hydrogen bonding, and hydrophobic forces can produce coacervates. ${ }^{88}$ From the molecular point of view, coacervates can also be formed by the interaction between BTAs and other polymers. ${ }^{89}$ Polyelectrolyte complex coacervates, coacervates formed by oppositely charged polymers, are of particular interest in the development of polymer DDSs for BTAs since nucleic acids, and proteins often carry negative charges. ${ }^{90}$ The morphologies of the resulting complex display a large diversity depending on the balance of water, polymer, and salt ions within the complex. ${ }^{91}$ In general, they can range from loosely associated colloids with more liquid-like properties in the case of coacervates to denser precipitates in the case of polyplexes. ${ }^{92}$

Synthesis. In solution, polyelectrolytes are attracted to oppositely charged materials and interfaces and exhibit a transition to an adsorbed state, resulting in nanospheres with physical crosslinking between the polymer and the BTA (Fig. 3a). ${ }^{93}$ Such complexation with polymers allows the drug to overcome critical extracellular and intracellular barriers in order to perform its specific biological function at the targeted place. ${ }^{94}$ Coacervates and polyplexes are usually prepared by mixing two polymer solutions through pipetting followed by maturation during a short incubation time. ${ }^{95}$ Because of the labile nature of the resulting interactions, the complex formation is dynamic. It can be reversed in response to small changes in local conditions. ${ }^{90}$ In such cases, various parameters such as the ionic strength, the ratio of polyacid to polybase, the total polyelectrolyte concentration and molecular weight, the $\mathrm{pH}$, as well as the temperature, play major roles. ${ }^{96}$

Many studies have investigated the influence of the polyanion/polycation ratio. For example, polyplexes prepared from DNA and poly-L-lysine showed that the highest DNA loading occurs at a DNA/poly-L-lysine mass concentration ratio between two to three. ${ }^{97}$ Other studies using PDMAEA or chitosan for the complexation of DNA showed similar results, suggesting that increasing the DNA/polymer ratio leads to the formation of durable complexes and provides a better coverage as well as a better release of DNA. ${ }^{98,99}$ Another key parameter in the formation of the polyplexes is the molecular mass of the polymer employed. Studies have investigated the influence of the molecular mass of polyethylenimine (PEI) on its complexation performance. PEI is one of the most widely employed polymers for the formation of polyplexes. It was shown that high molecular weight PEI (800 kDa) forms significantly more compact and more stable complexes with DNA and leads to an increased transfection efficiency compared to PEI with a lower molecular weight. However, the cell viability simultaneously decreased with the increasing molecular weight of the PEI, and a very tight complexation of the DNA by the PEI can also hinder the efficient intracellular release. ${ }^{59}$ Hence, a balance between high complexation efficiency, controlled dissociation at the targeted site, and low toxicity for PEI/DNA complex can be struck by tuning the molecular weight of the PEI. Furthermore, the ideal molecular weight for the complexation of one specific biomacromolecular therapeutic agent can differ from payload to payload. For example, the complexation and delivery of plasmid DNA, messenger RNA (mRNA), and replicon RNA (RepRNA) with a library of poly(2-ethyl-2-oxazoline)/poly(ethylene imine) copolymers with varying molar mass and charge densities showed that the optimal polymer design for each nucleic acid species was different and could significantly influence the transfection efficiency of the delivered nucleotide. ${ }^{100}$ Those BTAs 
a

\begin{tabular}{|c|}
\hline (Charged) \\
Biomacromolecular \\
Therapeutic Agent \\
\hline
\end{tabular}

b

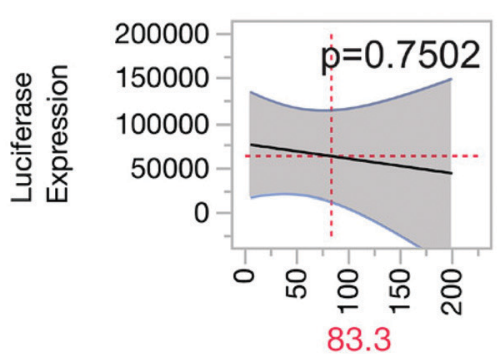

Polymer MW

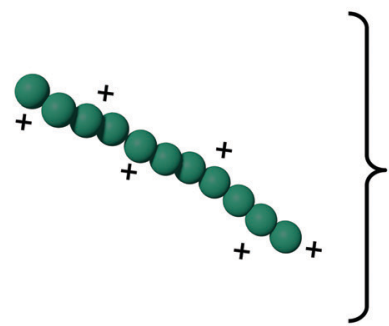

\section{Polymer or \\ Polyelectrolyte}

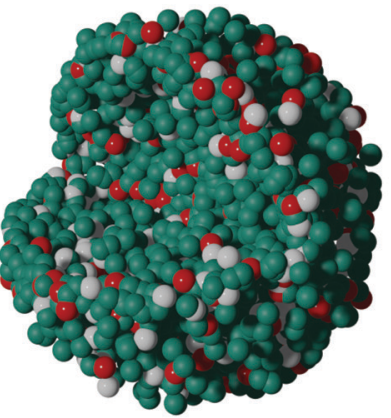

Coacervate / Polyplex

\section{DNA}

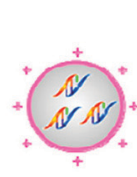

siRNA/ AD SiRNA/AD
polyplex

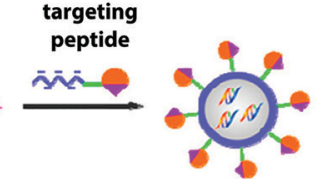

siRNA/ AD/peptide polyplex
mRNA

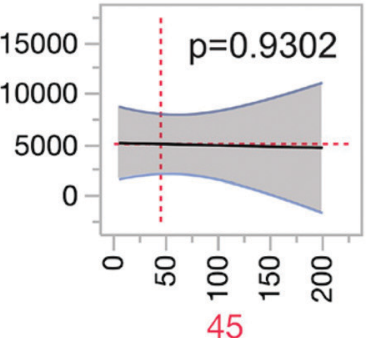

Polymer MW

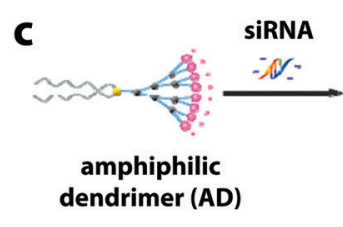
dendrimer (AD)

\begin{abstract}
(n)
\end{abstract}

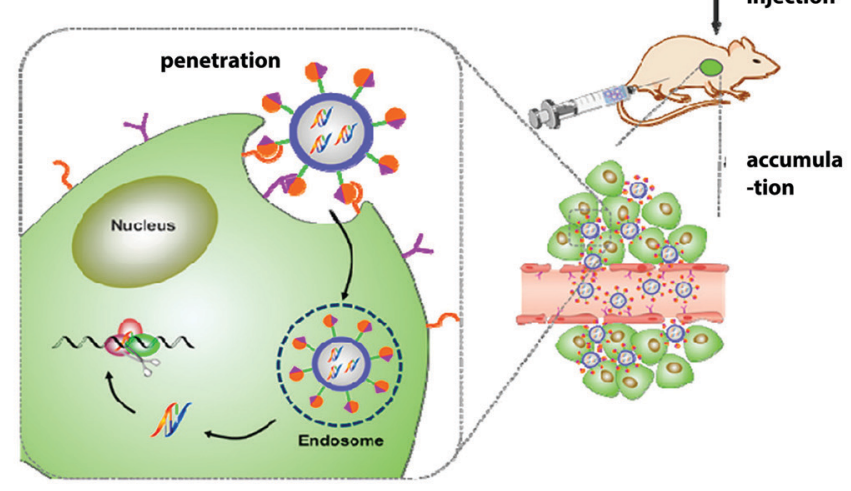

d

\section{RepRNA}

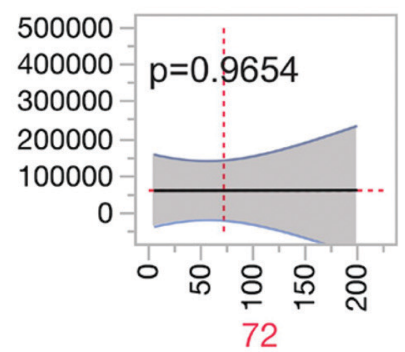

Polymer MW

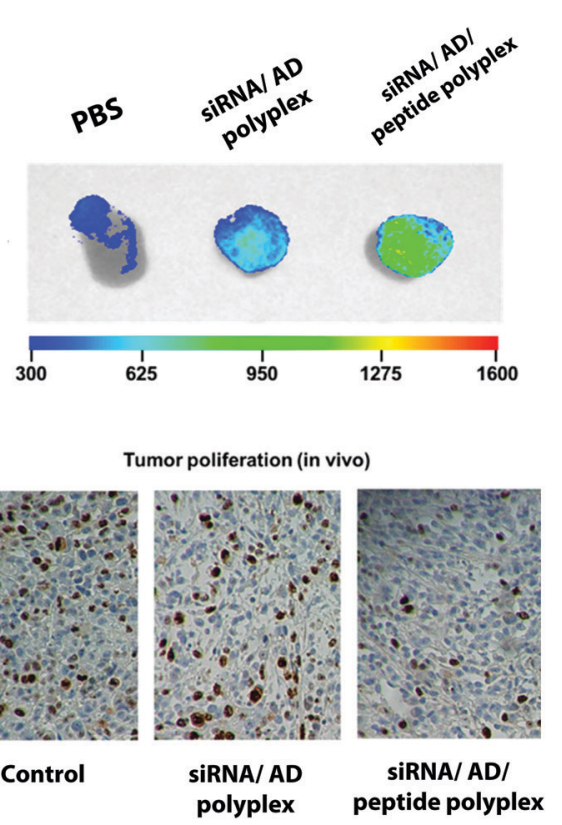

Fig. 3 (a) Schematic representation of the formation of polyplexes, (b) prediction profiles for the optimal polymer molecular weight for different type of nucleic acid materials, with luciferase expression as the output. Gray shading indicates $95 \%$ confidence interval, and the $p$-value indicates statistical significance, ${ }^{100}$ (c) illustration of the formation of targeted complexes for siRNA delivery using an amphiphilic dendrimer (AD) and the targeting peptide $E_{16} G_{6} R G D K, 60$ (d) targeted siRNA complexes (siRNA/AD/E ${ }_{16} G_{6} R G D K$ ) accumulate more efficiently in tumor tissues from mice than non-targeted polyplexes (siRNA/AD), ${ }^{60}$ (e) targeted siRNA/AD/ $E_{16} G_{6} R G D K$ polyplexes are much more potent than the non-targeted siRNA/AD systems for anti-cancer activity in mice tumors: cancer cell proliferation in tumor tissue revealed by immunohistochemistry using Ki67 staining (brown-stained cells indicate Ki67 protein expression correlating with metastasis). ${ }^{60}$ (b) Reprinted with permission from ref. 100, Copyright 2018 American Chemical Society; (c-e) reprinted with permission from ref. 60. Copyright 2018 American Chemical Society.

differ significantly in their structural composition and charge density, and it was found that the polymer molecular weight forming the most efficient polyplexes decreased from 83,72 , and $45 \mathrm{kDa}$ for DNA, RepRNA, and mRNA complexation (Fig. 3b). 
Many studies have investigated the influence of the polyanion/polycation ratio. For example, polyplexes prepared from DNA and poly-L-lysine showed that the highest DNA loading occurs at a DNA/poly-L-lysine mass concentration ratio between two to three. ${ }^{97}$ Other studies using PDMAEA or chitosan for the complexation of DNA showed similar results, suggesting that increasing the DNA/polymer ratio leads to the formation of durable complexes and provides a better coverage as well as a better release of DNA. ${ }^{98,99}$ Another key parameter in the formation of the polyplexes is the molecular mass of the polymer employed. Studies have investigated the influence of the molecular mass of polyethylenimine (PEI) on its complexation performance. PEI is one of the most widely employed polymers for the formation of polyplexes. It was shown that high molecular weight PEI (800 kDa) forms significantly more compact and more stable complexes with DNA and leads to an increased transfection efficiency compared to PEI with a lower molecular weight. However, the cell viability simultaneously decreased with the increasing molecular weight of the PEI, and a very tight complexation of the DNA by the PEI can also hinder the efficient intracellular release. ${ }^{59}$ Hence, a balance between high complexation efficiency, controlled dissociation at the targeted site, and low toxicity for PEI/DNA complex can be struck by tuning the molecular weight of the PEI. Furthermore, the ideal molecular weight for the complexation of one specific biomacromolecular therapeutic agent can differ from payload to payload. For example, the complexation and delivery of plasmid DNA, messenger RNA (mRNA), and replicon RNA (RepRNA) with a library of poly(2-ethyl-2-oxazoline)/poly(ethylene imine) copolymers with varying molar mass and charge densities showed that the optimal polymer design for each nucleic acid species was different and could significantly influence the transfection efficiency of the delivered nucleotide. ${ }^{100}$ Those BTAs differ significantly in their structural composition and charge density, and it was found that the polymer molecular weight forming the most efficient polyplexes decreased from 83, 72, and $45 \mathrm{kDa}$ for DNA, RepRNA, and mRNA complexation (Fig. 3b).

Examples of application. The complexation of BTAs with polymers can also involve additional materials. In this regard, polyplexes are able to deliver complex therapeutic agents to specific cancer cells by including active targeting. The targeting moiety attached to the polyplex may interact with ligands and receptors overexpressed on cancer cells. This enables the controlled delivery of the therapeutic agents specifically to the diseased cells and reduces potential collateral damage to normal tissue. ${ }^{101}$ For instance, dendrimer-based polyplexes using a amphiphilic dendrimer (AD) were designed to bind efficiently, by electrostatic interactions, both siRNA used as a therapeutic agent and a cell-targeting peptide $\left(\mathrm{E}_{16} \mathrm{G}_{6} \mathrm{RGDK}\right)$ to target a specific cancer cell line (Fig. 3c). In this system, the peptide on the surface of the polyplexes targeted the tumor endothelium by interacting with $\alpha_{\nu} \beta_{3}$ integrin, which is overexpressed in tumor vasculature. Furthermore, it also binds to the neuropilin-1 (Nrp-1) receptor present on tumor cells, hence promoting cancer cell penetration and uptake (Fig. 3d). ${ }^{60}$
Such a rational design of the multicomponent polyplex resulted in higher mean fluorescence intensities in tumors for the targeted system siRNA/AD/ $\mathrm{E}_{16} \mathrm{G}_{6} \mathrm{RGDK}$ when compared to the nontargeted system siRNA/AD (Fig. 3d). Consequently, the functionalization of the drug delivery system with the targeting peptide significantly enhanced the in vivo tumor targeting ability of the polyplexes. Following immunohistochemistry analysis showed that the proliferation of the cancer cells decreased more efficiently in mice tumors after the application of the targeted siRNA/AD/ $\mathrm{E}_{16} \mathrm{G}_{6}$ RGDK polyplexes when compared with nontargeted systems (Fig. 3e).

Polyplex gene delivery has lately also advanced to clinical trials. One of the first examples of a systemic administration of polyplex therapeutics applied to humans was a cationic cyclodextrin-based polyplex developed for the delivery of siRNA, which can reduce the expression of a ribonucleotide reductase and hence decreases tumor growth. ${ }^{63}$ In the phase I clinical trial 24 patients with different cancers were treated with the polyplex formulation and the clinical safety of the therapy was evaluated. The results showed that the formulation was well tolerated and the delivery system enabled a targeted delivery of the siRNA.

A currently ongoing phase II clinical trial (NCT02806687) is evaluating the ability of linear PEI complexed with plasmids encoding genes to achieve intratumoral gene transfer and to sensitize cancer cells to gemcitabine for pancreatic adenocarcinoma therapy. ${ }^{62}$ Phase 1 of the trial with 22 patients has demonstrated that the formulation can inhibit primary-tumor progression and dissemination when combined with additionally administered gemcitabine. Currently, the study measures the clinical benefits of the therapy, such as a progression-free survival and tumor response, the biological tumor markers, the biodistribution, and quality of life change in 100 patients with locally advanced tumors. ${ }^{102,103}$

Advantages and limitations. The main advantage of the complexation of BTAs with polymers in coacervates and polyplexes is the absence of a chemical modification of the therapeutic agent. ${ }^{104}$ Hence, the sensitive intermolecular interactions in the molecule can be more easily preserved and are not hampered by external chemical modifications. This leads to improved preservation of the intrinsic bioactivity of the drug after the complexation in comparison to polymer functionalization. The formation of the polyplex can protect and facilitate the cellular uptake of the cargo. Moreover, especially in the case of charged polyplexes with positive surface potential, the polyplexes can efficiently improve the internalization of their payloads by the cells because they interact electrostatically with the negatively charged cell membranes and mediate the endosomal membrane destabilization required for the cytosolic release and delivery at the intended site of action. ${ }^{105}$ The excellent delivery of the cargo in the polyplex to the cytoplasm is often ascribed to the so-called proton sponge effect of cationic material where the endosomal membrane destabilization is caused by the inflow of anionic molecules for charge compensation. ${ }^{106,107}$ This results in the swelling and generation of osmotic pressure in the endosomal compartment and 
leads to the disruption of the membrane. ${ }^{108}$ This process strongly improves the polyplex delivery efficiency and is another significant advantage of such DDSs.

However, this approach is also constrained by some factors. While gene materials are usually strongly charged and thus very suitable for the formation of polyplexes, the formation of polyplexes with certain proteins and polypeptides, which can be moderately charged or even neutral at physiological conditions depending on their isoelectric point, can be challenging. ${ }^{109}$ Additionally, the number of charged residues in a protein is limited and strongly varies from one protein to another; this can further hamper the formation of proteinbased polyplexes. ${ }^{110}$ More generally, the systemic delivery of polyplexes can be hindered by their instability under physiological conditions. Indeed, various physiological salt and proteins found in blood can readily bind to the polyplexes and increase the risk for the premature release of the payload or even for the complete dissociation of the polyplex. ${ }^{111}$

\subsection{Layer-by-layer assemblies}

Another class of drug delivery platforms for BTAs based on electrostatic complexation is the layer-by-layer (LbL) assembly of polyelectrolytes. This method expands the simple electrostatic complexation by the design of multilayer architectures with layers of, usually, alternating charges with nanometer-scale precision. The technique is based on the alternating adsorption of complementary layers of building blocks, which can be synthetic or natural polyelectrolytes and BTAs (Fig. 4a). ${ }^{112,113}$ For applications in drug delivery, colloidal LbL particles and capsules are of particular interest. In these cases, the single layers can be assembled either on a core template, which can be a drug reservoir or a solid nanoparticle. ${ }^{114}$ To form an LbL particle system, a colloidal substrate bearing a defined charge or a polyelectrolyte in solution is mixed with complementary polyelectrolytes followed by washing, usually through centrifugation. ${ }^{115}$ These steps can be repeated several times, each time using an oppositely charged macromolecule to the charge of the previous step to achieve the desired loading of the BTA (Fig. 4b).

Synthesis. Similarly to the formation of electrostatic polyplexes, the LbL deposition has the advantage of using mild conditions (aqueous solutions, absence of chemical modifications) that are favorable for preserving the fragile structure and activity of the BTAs. Once covered by multiple layers of polyelectrolytes, the cargo is protected, and the risk of degradation during transport decreases. ${ }^{60,111,112,113}$

LbL formulations used in the delivery of BTAs allow for controlled loading of the nanocarrier with the biomacromolecule. Since the LbL assembly of the BTA can be monitored, for instance, on a silicon oxide-coated quartz sensor as found in a quartz crystal microbalance with dissipation, the exact amount of adsorbed therapeutic agents can be calculated. ${ }^{116}$ In this regard, LbL assemblies can serve as a precise gene delivery platform. For example, this led to LbL systems being used to load a plasmid DNA encoding a green fluorescent protein (GFP) in defined quantities. The plasmid DNA was complexed on the surface of PLGA nanoparticles in combination with poly(L-lysine) (PLL) and poly( $\gamma$-glutamate) (PGA) via the LbL technique. A defined amount of $0.5 \mu \mathrm{g}$ of the plasmid DNA in the LbL assemblies was loaded during the synthesis process. After the formulation of the plasmid vector in the LbL assembly, the gene delivery efficacy was tested by the expression of GFP in HEK293 cells. This DDS was compared to Lipofectamine, a commercial gene delivery system, loaded with $2 \mu \mathrm{g}$ of GFP encoding plasmid DNA. No significant differences were observed for the transfection efficiency of the GFP expressing plasmid in HEK293 cells when using the LbL DDS or the Lipofectamine containing 4 times more plasmid. Hence, the LbL assemblies can deliver the plasmids at the same efficiency as Lipofectamine but require less plasmid material and also cause lower cytotoxic effects in HEK293 cells than the Lipofectamine. ${ }^{116}$

Examples of application. In another study, LbL assemblies were used to deliver siRNA that knocks down a drug-resistance pathway in tumor cells. This LbL nanosystem was prepared by the layering of siRNA and poly-L-arginine (pLArg). ${ }^{65}$ In a quantitative manner, roughly 3500 siRNA molecules could be loaded in a single layer and the surface of the LbL assemblies was further coated by a layer of hyaluronic acid, which has been previously reported to enhance in vivo stability (Fig. 4c). First, to evaluate the pharmacokinetics of the LbL nanoparticles, AlexaFluor677 dye-labeled nanoparticles were administered to mice via the tail vein. A fluorescence imaging experiment of the whole mouse confirmed the stability of the assemblies, as they circulated in the animal for 12 days and showed low levels of liver clearance (Fig. 4d). Then, nude mice bearing luciferase-expressing subcutaneous xenografts received luciferasetargeting siRNA LbL DDSs in a single dose of $1.4 \mathrm{mg} \mathrm{kg}^{-1}$ to induce the knockdown after an intravenous systemic administration. After 5 days post-injection the analysis of the tumor tissues showed a 4-fold decrease in luciferase mRNA levels, revealing a sustained delivery and a therapeutic effect of the siRNA in the mice (Fig. 4e).

The LbL system can be built by integrating a variety of functions and molecules in the different layers. For instance, multiple BTAs and adjuvants can be easily incorporated in LbL systems. One therapeutic agent can be deposited in the core of the assembly and another one in the surrounding layers, thus creating an independently tunable device for the co-delivery of multiple therapeutic agents. ${ }^{65}$ This co-localization of drugs enables the construction of defined signal pathways and artificial architectures that can mimic biological functions found in natural organisms. ${ }^{118}$ For example, polystyrene (PS) carriers were used as the substrate for the assembly of glucose oxidase (GOX) and horseradish peroxidase (HRP) in conjunction with PEI and polystyrene sulfonate. ${ }^{119}$ Because both enzymes preserved their enzymatic activity during the formation of the LbL nanosystems, coupled enzymatic reaction between the GOX and the HRP could still be performed after the assembly, and the LbL nanoparticles were able to mimic the sequential cascade reactions observed inside cellular compartments. ${ }^{120}$

Moreover, to increase the efficiency of immunotherapeutic treatments, it can be helpful to deliver simultaneously 
a

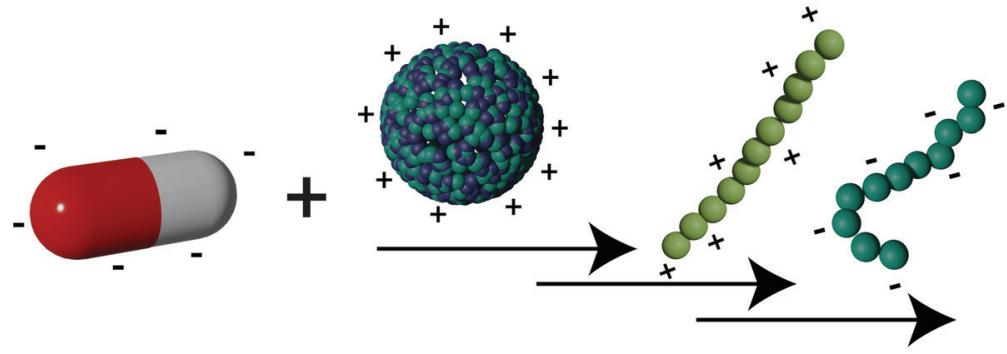

\section{(Multiple) Charged \\ Biomacromolecular Therapeutic Agent(s)}

b

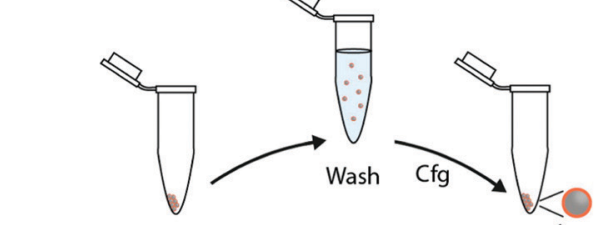

Polymer 1
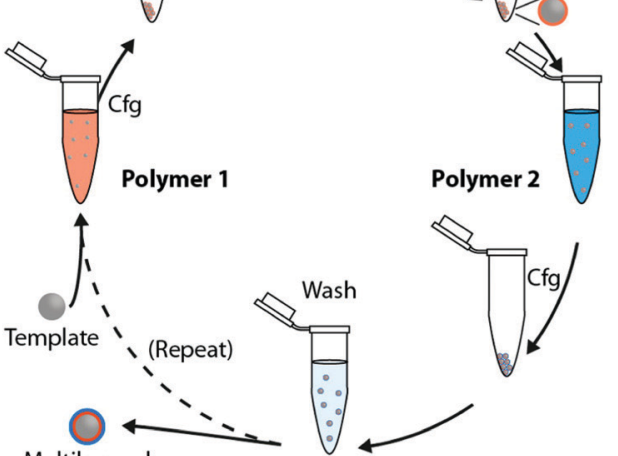

Multilayered particle

d
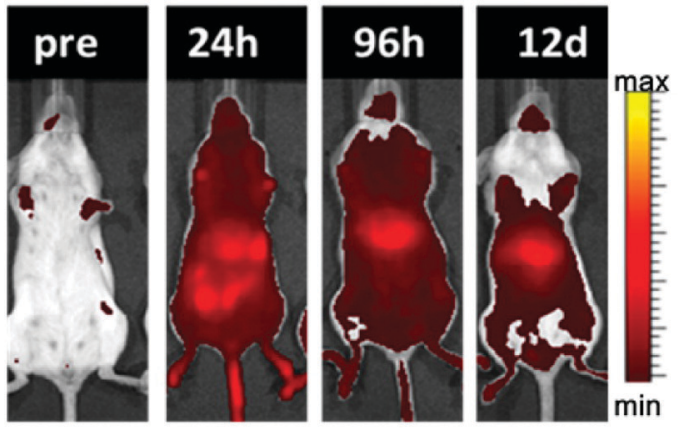

C

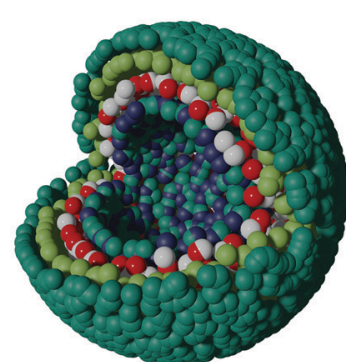

Layer-by-layer

Assembly
Nanoparticle core containing doxorubicin and other chemotherapy

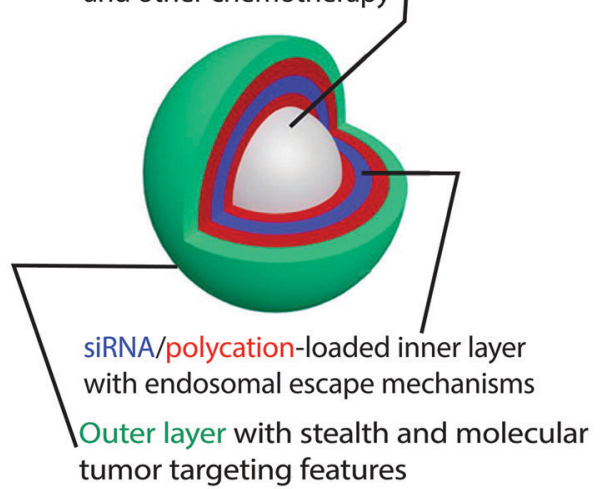

e

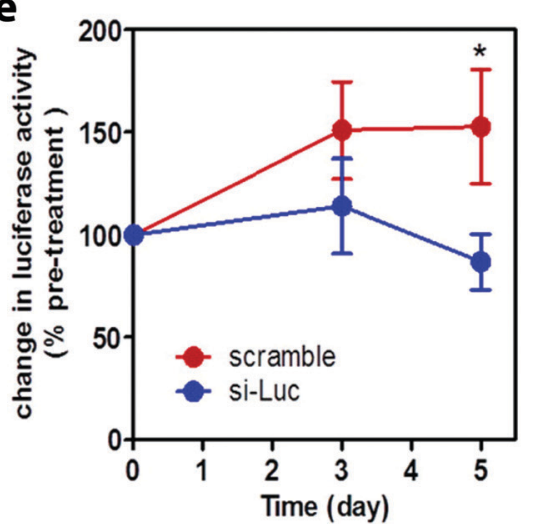

Fig. 4 (a) Schematic representation of layer-by-layer (LbL) assemblies, (b) schematic illustration of immersive assembly on particulate substrates using centrifugation (cfg) in between washing steps, ${ }^{117}$ (c) schematic representation of a siRNA drug delivery platform based on LbL assembly, ${ }^{65}$ (d) the LbL nanoparticle cores in (c) were fluorescently labeled with AlexaFluor677 to assess the biodistribution using live animal fluorescent imaging $(n=4)$, ${ }^{65}$ (e) systemic delivery of siRNA using LbL nanoparticles in mice models: bioluminescence activities in subcutaneous mice tumors treated with a single dose tail vein injection of $1.4 \mathrm{mg} \mathrm{kg}^{-1}$ of luciferase-targeting siRNA-loaded LbL assemblies (si-Luc) and siRNA control (scramble). ${ }^{65}$ (b) Reprinted with permission from ref. 117, Copyright 2014 The American Chemical Society; (c-e) reprinted with permission from ref. 65. Copyright 2013 The American Chemical Society.

multiple payloads. ${ }^{121}$ For example, in addition to the delivery of the biomacromolecular antigen, the co-delivery of adjuvant molecules is essential for the enhanced activation of immune cells. The flexibility of LbL structures has the potential to co-deliver a combination of antigen and adjuvant, even when they display diverse physicochemical properties in terms of molecular weight, hydrophilicity, and pharmacokinetics. ${ }^{122}$ LbL nanocarriers have been successfully used for such purpose due to their inherent ability to incorporate many active molecules in different layers within the same drug delivery system..$^{65,123,124}$ In one of 
such systems, ovalbumin, a model protein antigen, and an immune-activating single-stranded synthetic DNA adjuvant were combined in one LbL assembly enabling the co-delivery of two components to induce a strong immune activation in mice. ${ }^{64}$

For this purpose, ovalbumin was coated with two bilayers of dextran sulfate and pLArg. Subsequently, the immunogenic anionic DNA was complexed to the outer pLArg layer and coated with additional pLArg. The LbL nanosystems loaded with ovalbumin and immune-activating DNA were readily internalized by dendritic cells. Furthermore, they promoted a strong upregulation of co-stimulatory markers combined with a vast secretion of pro-inflammatory and polarizing cytokines, whereas the OVA assemblies with dextran sulfate and pLArg lacking the DNA adjuvant totally failed to stimulate cytokine secretion. $^{64}$

Advantages and limitations. The variety of materials compatible with this approach is another advantage of this method. Many polyelectrolytes such as poly(allylamine hydrochloride), poly(diallyl dimethylammonium chloride), and PEI, poly(sodium styrenesulfonate), poly(sodium vinylsulfonate), and poly(acrylic acid) have been widely used for the synthesis of LbL assemblies. Moreover, since many BTAs are charged, they are also optimal candidates for the assembly by electrostatic LbL. Unfortunately, the LbL systems, just like the polyplexes, can suffer from inherently limited stability in vivo due to the potential perturbation in the electrostatically assembled systems. ${ }^{124}$

\subsection{Polymer micelles and polymersomes}

Polymer micelles and polymersomes are supramolecular nanostructures usually formed by the self-assembly of amphiphilic block copolymers. ${ }^{125}$ Classically, they are composed of block copolymers containing both hydrophilic and hydrophobic segments, and both polymer micelles and polymersome can be obtained from diblock, triblock or multiblock copolymers. ${ }^{126,127}$ In aqueous environments, the block copolymers assemble into nanostructures depending on the polymer composition. The different assemblies result from the relative volume occupied by the hydrophilic(s) and hydrophobic(s) blocks. This defines the geometric packing of block copolymers in the resulting copolymer assemblies in aqueous solution and controls the shape of the resulting assembly. For example, a diblock copolymer composed of a hydrophilic and a hydrophobic block can either assemble into spherical micelles, worm-like micelles, or vesicles (or polymersomes) as the length of the hydrophobic block increases. $^{128}$

In polymer micelles used for the delivery of BTAs, the assembly usually occurs in water, and the hydrophobic part of the block copolymer is excluded from the aqueous surrounding forming the micelle core. It is in this hydrophobic pocket at the center of the micelle that payloads, like BTAs, are typically encapsulated. The hydrophilic part of the block copolymer then builds the nanoparticle shell with a brush-like architecture (Fig. 5a).

Polymersomes made from diblock copolymers self-assemble in a bilayer polymer membrane ${ }^{129}$ reminiscent of the phospholipidic double layer of the liposome. The block copolymers used can be considered synthetic mimics of phospholipid to some extent. ${ }^{130}$ Typically, the polymersomes used in the delivery of BTAs have an aqueous core separated from the surrounding aqueous environment by a semi-permeable hydrophobic membrane (Fig. 5a). In the case of polymersomes, the payloads can be located either in the hydrophobic membrane or inside the hydrophilic core, providing more versatility to encapsulate a wide variety of payloads. ${ }^{129}$

Synthesis. In both micelles and polymersomes, non-covalent intermolecular interactions mainly drive the formation of the nanocarrier. If amphiphilic polymers assemble, solvent molecules are released because the hydrophobic components are withdrawn from the aqueous media leading to an overall increase in the entropy of the system. An important parameter for the construction of self-assembled polymer micelles is their stability against dissociation, especially under the highly diluted conditions that can be observed after systemic injection. This parameter is described by the critical micelle concentration (CMC) ${ }^{131}$ Below the CMC, the amphiphilic polymers only exist as single individual chains. As the polymer concentration increases and reaches the CMC, the polymer chains start to associate and form micelles. In comparison to lowmolecular-weight surfactants, block copolymers generally exhibit a lower CMC and thus higher stability. This property is attributed to the larger polymer segments resulting in stronger interactions. For instance, the CMC of block copolymers is typically between $0.0005-0.002 \%,{ }^{131}$ while the CMC of small molecules surfactants like sodium dodecyl sulfate is $c a .0 .2 \%$. Amphiphiles with higher CMC values may not be suitable in the design of DDSs since they are unstable in a dilute aqueous environment and easily dissociate due to the strong dilution experienced following administration.

However, the formation of the self-assembled structure, such as micelles and polymersomes, is not always straightforward. Different methods exist to convert a block copolymer solution into a suspension of self-assembled structures. ${ }^{126}$ Some of the most frequently used strategies involve a "solvent exchange". The block copolymer is first dissolved in an organic solvent, which is chosen because it is a good solvent for all the blocks, and then the solution is transferred to the selective solvent, usually water when micelles and polymersome for the encapsulation of BTAs are prepared. The solution of block copolymer in the organic solvent can be added directly to water under vigorous stirring, slowly dialyzed against water, or water can be added in a step-wise manner to the organic solution. The resulting suspension is then extensively dialyzed against water to remove any traces of the organic solvent. ${ }^{132}$ Following the formation of the polymer aggregate, the extrusion of the resulting dispersion through nanoscale pores leads to uniformization of the size and may also be used to induce the transition from spherical to cylindrical geometries in the case of micelles. ${ }^{133}$ However, the formation of large polymersomes can be challenging, and more complex formation protocols need to be implemented. ${ }^{134}$

Most micelles and polymersomes are built through the delicate balance of hydrophilic/hydrophobic segments. Interestingly, the 
a
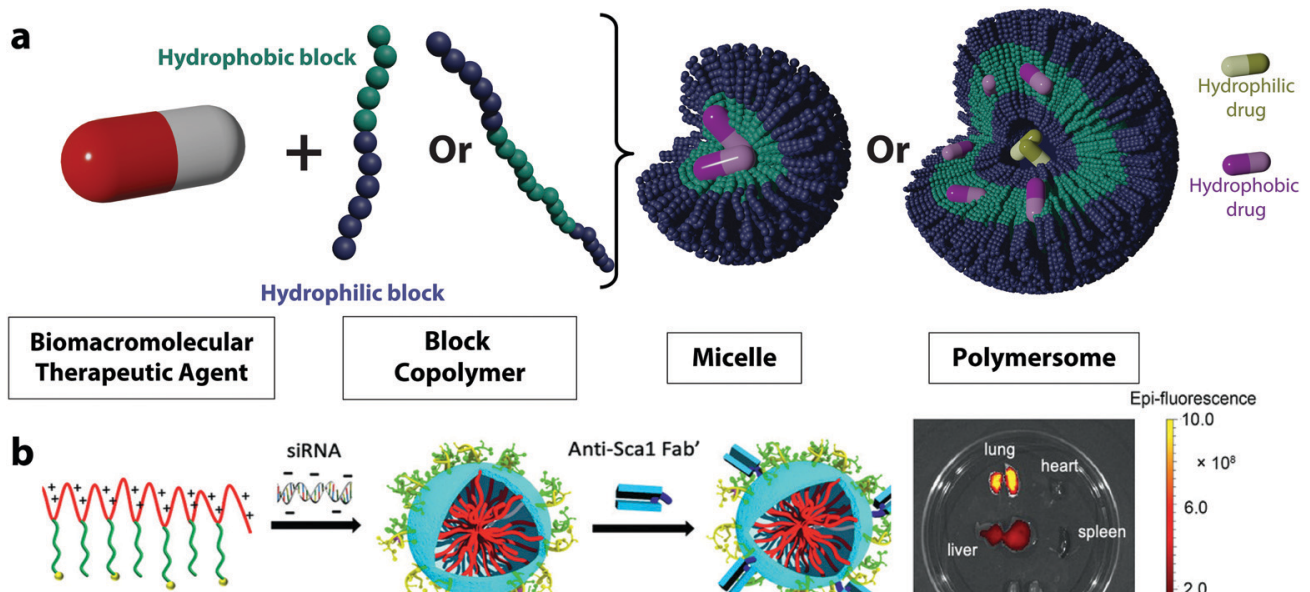

Block Copolymer
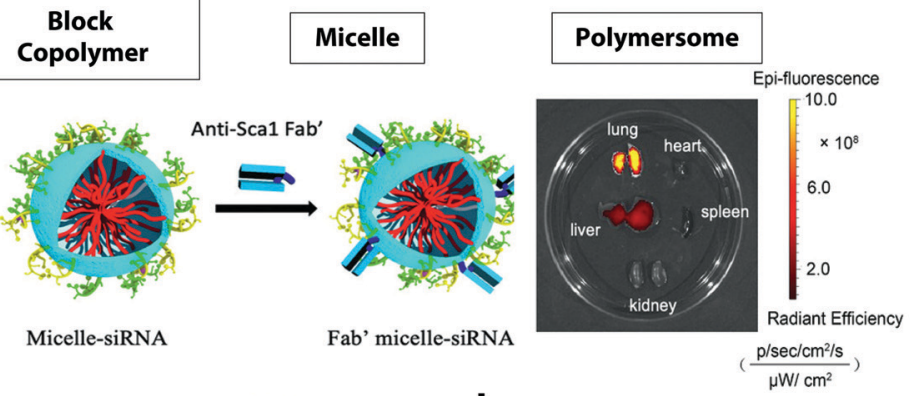

C

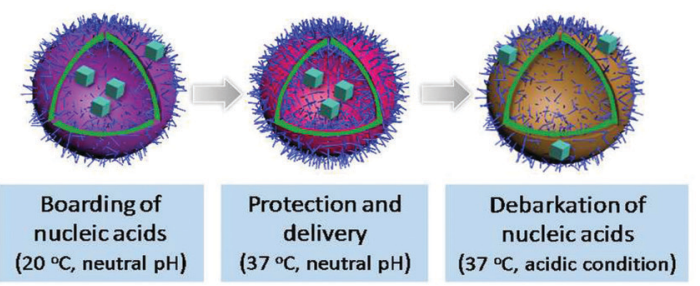

d
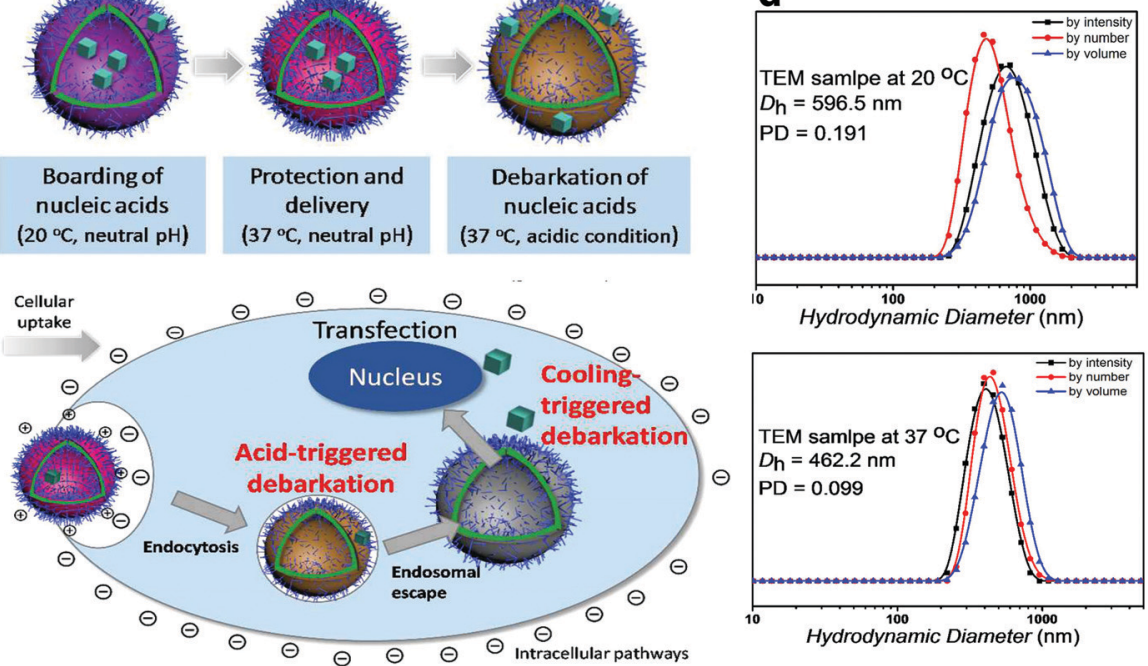

Hydrodynamic Diameter $\stackrel{1000}{100}$

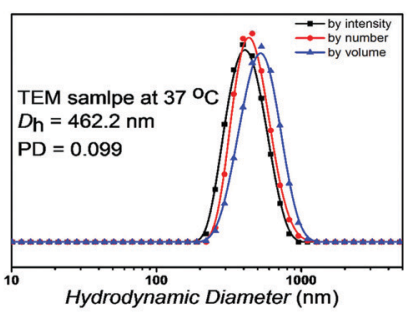

e

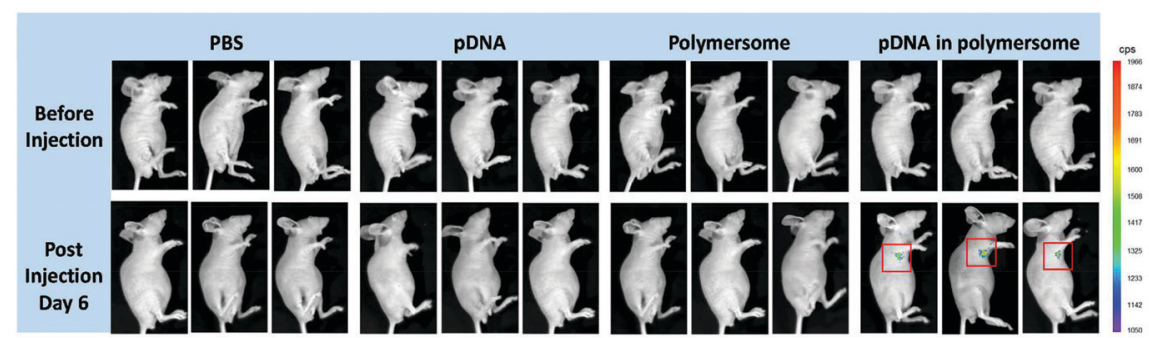

Fig. 5 (a) Schematic representation of polymeric micelles and polymersomes, (b) scheme of the formation of siRNA-loaded micelles via the assembly of negatively charged siRNA and the positively charged block copolymers PEI-g(20)-PEG-MAL, followed by the conjugation of a stem cell antigen 1 antibody (Anti-sca $1 \mathrm{Fab}^{\prime}$ ) for the targeting of lung mesenchymal stem cells and Alexa 647 fluorescence images of the organs of a mouse administrated with Fab'-conjugated Micelle, ${ }^{67}$ (c) self-assembly of polyNIPAM-polyDEA block copolymers into a polymersome with a "boarding gate" and a "release gate", ${ }^{66}$ (d) DLS measurements of crosslinked polymersomes at different temperatures, ${ }^{66}$ (e) in vivo transfection and expression of plasmid DNA delivered by polymersomes and controls (experiments conducted with triplicates). ${ }^{66}$ (b) Reprinted with permission from ref. 67. Copyright 2021 Wiley, (c-e) reprinted with permission from ref. 66. Copyright 2018 The American Chemical Society.

interaction of the block copolymer with the BTAs can itself be used as the driving force for the formation of micelles. The complexation of a charged BTA and an oppositely charged polymer can lead to the formation of a polyplex (Section 3.2). However, when the polymer used is a block copolymer composed of a poly(ionomer) segment and a neutral hydrophilic segment, the loss of hydration is associated with the formation of the ionic complex and the neutralization of the charged polymer block leads to the formation of polyion complex micelles. In such a case, the hydrophobic segment is the complex of the ionomer block of 
the copolymer and the BTA, which is then surrounded by the neutral hydrophilic block. The neutralization of the charge of the block copolymers by a charged payload induces the required amphiphilicity for the micelle assembly. ${ }^{135,136}$

The encapsulation of the payload often occurs through a passive loading mechanism for both polymer micelles and polymersomes. ${ }^{137}$ The hydrophilic BTAis embedded in the inner aqueous core of the polymersome or a hydrophobic agent can be encapsulated in the solid core of the micelles during the nanocarrier formation. The loading efficiency of this method is usually low, and although different studies successfully encapsulated oxygen transport proteins, like hemoglobin and myoglobin, into polymersomes while retaining their bioactivity, the encapsulation efficiencies did not exceed $30 \% .{ }^{138-140}$ Inversely, the use of active loading procedures, where the loading usually takes advantage of diffusion properties following a gradient of concentration across the bilayers, results in a payload encapsulation with improved efficiency compared to passive methods. However, the efficiency of this method is impeded by the large hydrodynamic radius of BTAs, which results in low diffusivity of the molecules across the bilayer structures.

Examples of application. The flexibility to combine several polymer segments in one polymer chain allows for the development of multifunctional micellar delivery systems and custom-made architectures. One interesting approach includes the synthesis of micelles composed of branched polyethylenimine modified with PEG. The micelles were used for the delivery of a transcription factor-1 small interfering RNA (siRNA) to the lung (Fig. 5b). ${ }^{67}$ Such a treatment aims at inhibiting the myofibroblast differentiation of lung-resident mesenchymal stem cells for pulmonary fibrosis therapy. The copolymers of cationic PEI grafted with maleimide-PEG (PEI- $g$-PEG-Mal) successfully encapsulated the siRNA and their surface was further modified with a stem cell antigen 1 antibody (anti-Sca1 Fab') to target mesenchymal stem cells in the lung (Fig. 5b). The siRNA-loaded micelles modified with anti-Sca1 Fab' preferentially accumulated in the lung and were able to suppress the myofibroblast differentiation of lung-resident mesenchymal stem cells because they ablated the expression of a RUNX1 gene. In a similar approach, copolymers of cationic poly[ $N^{\prime}-[N$-(2-aminoethyl)-2aminoehtyl]aspartamide] and PEG were able to electrostatically bind mRNA and further self-assemble into micelles. ${ }^{68}$ This micellar system has proceeded to animal studies for the treatment of Alzheimer's. Alzheimer's disease is characterized by an imbalance between the synthesis and degradation of amyloid-beta peptides in neural cells. The degradation of the peptides can be controlled by protein neprilysin. The delivery of mRNA encoding neprilysin could allow to decrease amyloid-beta deposition and prevent the pathogenic changes occurring in the brain. Animal studies have revealed that the administration of this micellar system containing the mRNA increased the level of neprilysin and consequently reduced the concentration of amyloid-beta peptides. The success of the micellar formulation can be ascribed to the fact that it prevented the digestion of the mRNA by nucleases and the recognition of the biomacromolecule by the immune system, both of which increased the efficiency of the delivery of the mRNA to the cells.

Polymersome DDSs for the delivery of BTAs have entered various preclinical testings. For instance, polymersomes prepared from a poly(ethylene glycol)- $b$-poly(dithiolane trimethylene carbonate-co-trimethylene carbonate)- $b$-polyethylenimine triblock copolymers were functionalized with apolipoprotein $\mathrm{E}$ derived peptides showing an excellent ability to cross the blood-brain barrier and aiming at the treatment of glioblastoma, a primary tumor inside the brain that still remains incurable. ${ }^{141}$ The polymersomes loaded with the therapeutic protein saporin displayed a highly specific and potent antitumor response for orthotopic human glioblastoma xenografts in animal studies. In another very recent study, polymersomes composed of polybutadiene- $b$ polyethylene glycol and 1,2-dioleoyl-3-trimethylammoniumpropane block copolymers were developed to co-deliver the SARS-CoV-2 spike protein and a DNA nucleotide immunestimulatory adjuvant as a vaccine against SARS-CoV-2. ${ }^{69}$ The animal study revealed that two doses of the polymersome formulation induced neutralizing antibody titers in mice for up to 40 days, while the co-administration of the adjuvant reduced the required dosage of the SARS-CoV-2 spike protein.

In polymersomes, the incorporation of functional polymer segments in the polymer chain allows for the design of smart membranes. The release of the encapsulated molecules can then be controlled by the functionalization of the membrane with molecules tuning the permeability of the membrane and increasing the mass transport of the active molecules. When dealing with macromolecular therapeutic agents, it is especially crucial to increase the permeability of the membrane. ${ }^{66}$ Several approaches have been developed to do so, such as the functionalization of the polymersome membrane with channel-forming transmembrane proteins, ${ }^{142}$ DNA nano-pores, ${ }^{143}$ or smart gates. ${ }^{66}$ For instance, inspired by biological cell membranes, where various proteins serve for ion transport, polymersomes with selective membrane permeability were designed by introducing membrane proteins, such as aquaporin ionomycin in the membrane shell. ${ }^{144,145}$ For this purpose, the aquaporin, a natural membrane protein forming pores due to their assembly as trimers, can be mixed with the block copolymers prior to the self-assembly. After the formation of the polymersome, the proteins incorporated in the membrane allow for a selective release of molecules. ${ }^{142}$ DNA nanopores provide another possibility for the controlled transport across polymersome membranes. ${ }^{146}$ They can be fabricated through the selfassembly of oligonucleotides, and they can build controlled holes in the polymer layer. ${ }^{143}$ They have been integrated into polymersomes by incubating the polymersomes with DNA fragments after their synthesis. The DNA pores insert into the membrane, first by tethering to the membrane, followed by complete insertion depending on the concentration of the oligonucleotides. The resulting nanopores act as gates controlling the selective release of payloads based on their size. ${ }^{143}$

However, the elaborate engineering of the polymersome membranes successfully leads to the controlled release of small molecules and drugs, but the release of biomacromolecules 
through polymersome pores is one of the current challenges. The design of smart membranes can provide one solution to this, as it can incorporate heterogeneous membrane sections with finely controllable and responsive behavior. ${ }^{66}$ By incorporating responsive polymers in the membrane, the permeability of the membrane can be controlled. The polymers used in such cases can adjust their properties in response to external stimuli which can lead to the swelling of the membrane and the controlled release of payload. One exemplary system forming smart gates in polymersome membranes exploits the responsive polymers polyNIPAM and poly(diethylamino)ethyl methacrylate (polyDEA). ${ }^{66}$ After the self-assembly in a mixture of water and tetrahydrofuran, the thermoresponsive polyNIPAM dehydrates and the pH-responsive polyDEA functionality is deprotonated. This results in polymersomes with closed gates in the membrane. The polyNIPAM-based segment enables the design of a "boarding gate" in the membrane, opening up below the LCST of PNIPAM $\left(20-30{ }^{\circ} \mathrm{C}\right)$. Under these conditions, biomacromolecules can be loaded, and the gate can be closed at physiological temperature $\left(37^{\circ} \mathrm{C}\right)$ to lock the payload (Fig. $5 \mathrm{c}$ ). The pH-sensitive polyDEA segments in the polymersome membrane were designed to serve as the "release gate", allowing the release of the encapsulated plasmid DNA in acidic environment (such as in the endosomes) upon the protonation of the polyDEA. The protonation leads to a swelling of the polymersomes from around $450 \mathrm{~nm}$ at $\mathrm{pH} 6.8$ (Fig. 5d) to $610 \mathrm{~nm}$ at $\mathrm{pH}$ 5.4. Such polymersomes have been used for the transfection of cells with GFP-encoding plasmid DNA and the successful release of the payload in vitro and in vivo (Fig. 5e).

Advantages and limitations. The delivery of BTAs using polymer micelles and polymersomes provides a highly controlled platform for a broad range of therapeutic agents or a combination of therapeutic agents. The encapsulation of BTAs into polymersomes is a successful method for improving the half-life and overcoming physiological barriers while retaining the activity of the molecules. Modern processes to synthesize well-controlled and well-defined block copolymers allow the design of complex micelle structures with varying morphologies and compositions. The resulting micelles interact in different manners with biological systems, mainly due to their shape resulting in unique cellular uptake and targeting ability. Furthermore, the encapsulation of the macromolecular therapeutic agents does not require the use of harsh chemical modifications, and the dense polymer shell protects the therapeutic agents from denaturation. Unfortunately, similar to other self-assembled systems, micelles can suffer from inherently limited stability in vivo due to the potential perturbation in the non-covalent interactions governing the assembly. ${ }^{124}$

\subsection{Nanoparticles, nanogels, and nanocapsules}

Over the past decade, covalently crosslinked polymer nanocarriers have attracted growing interest for their use in the delivery BTAs due to their flexibility and adaptability in terms of hydrophilicity, stability, size, charge. ${ }^{147,148}$ Crosslinked polymer nanocarriers are nanosized networks composed of polymer chains linked together through chemical or physical crosslinking points
(Fig. 6a). In a polymer nanoparticle or polymer nanogel the polymer matrix is uniformly distributed in the entire nanocarriers, while in a nanocapsule, the polymer network is segregated at the carrier surface, creating a shell that can engulf either an aqueous or organic core. The main distinction between nanoparticles and nanogels is the swelling of the polymer network. In a nanoparticle, the defined three-dimensional polymer network is mostly desolvated, while in the case of nanogels, the network holds a large quantity of solvent. ${ }^{149}$

Synthesis. The crosslinked polymer nanocarriers used in the design of DDSs can be made from virtually any polymer, of natural or synthetic origin, or a combination of thereof, encompassing a wide range of chemical compositions and bulk physical properties. They can be produced by direct polymerization methods or made by the use of preformed polymers. ${ }^{25}$ They can be obtained from the crosslinking of self-assembled structures, solvent evaporation, polymerization in heterogeneous media like miniemulsion, precipitation polymerization, or many other techniques. ${ }^{24}$ Almost any polymerization reaction and any preformed polymer are suitable to be used in this vast range of process. For example, radical, anionic, cationic, oxidative or polyaddition polymerization reactions can be performed with many different monomers (e.g., styrene, acrylates, methacrylates, fluoroacrylates, acrylamides, aniline, pyrrole) to obtain solid polymer nanocarriers. ${ }^{150,151}$ Alternatively, physical interaction between preformed polymer can lead to the selfassembly of the polymers and can be used to produce nanogels. ${ }^{152-154}$ The crosslinking of the polymers in self-assembled systems is a powerful strategy to overcome the inherent risk of disassembly of self-assembled systems, which is often accompanied by the premature release of encapsulated payloads. Furthermore, if polymers are first self-assembled into vesicles with a hollow aqueous core based on hydrophobic or electrostatic interactions and further crosslinked, nanocapsules can be obtained. The crosslinking of self-assembled systems to produce nanocarriers often does not require the use of organic solvents or additional harsh reagents, which is advantageous for the encapsulation of highly sensitive BTAs. However, the prerequisite for this method is the use of polymers with both the ability to form polymer self-assemblies and the ability to be chemically crosslinked under mild conditions, which limits the number of suitable materials. ${ }^{155}$

Another technique for the preparation of nanoparticles is polymerization in heterogeneous media, like in a miniemulsion polymerization. ${ }^{156}$ When this method is applied to the encapsulation of BTAs, an aqueous solution of monomers, or already preformed polymers, is emulsified in a hydrophobic solvent, leading to an emulsion of water droplets in an oil phase stabilized by surfactants. ${ }^{157}$ The water droplets containing monomers or polymers are then polymerized or the preformed polymers are crosslinked to obtain solid polymer nanoparticles or nanogels. In contrast to the synthesis of nanoparticles and nanogels, nanocapsules can be obtained if the crosslinking agent is added to the continuous phase of the emulsion and that the reaction between the polymer and crosslinking agent occurs only at the interface of the two solvents (Fig. 6b). 

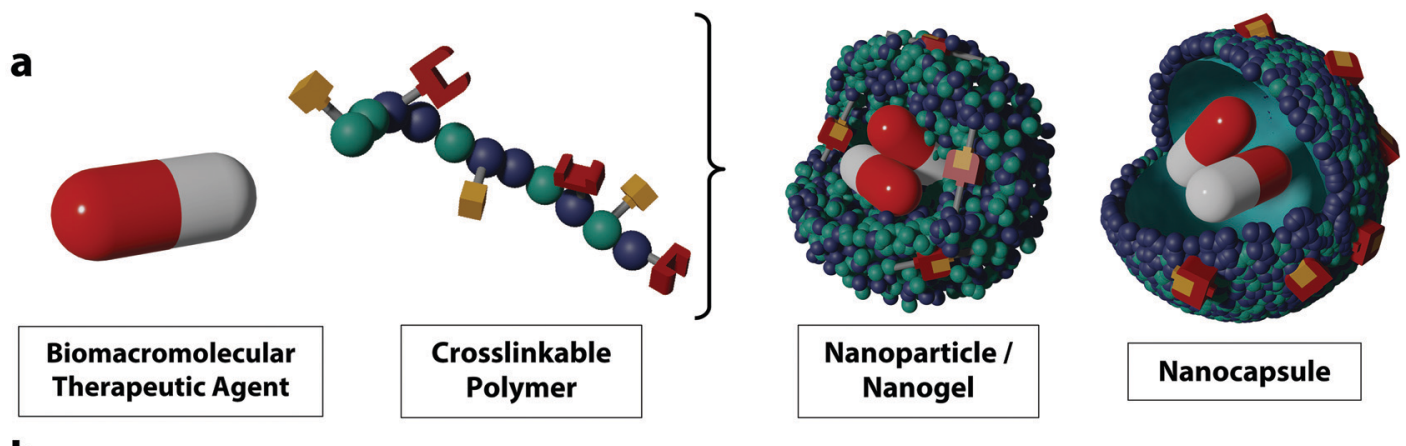

b
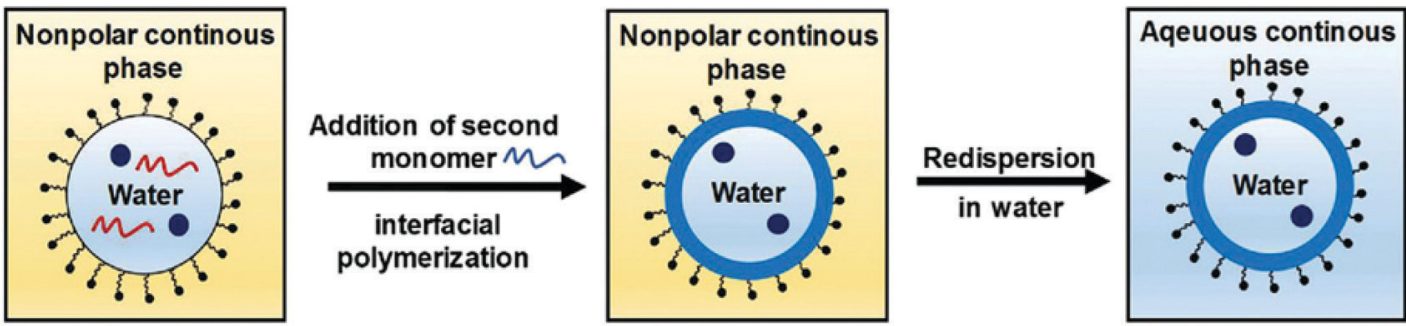

M - Hydrophilic monomer mo - Surfactant - Hydrophilic cargo

- Polymer Shell formed after poymerization
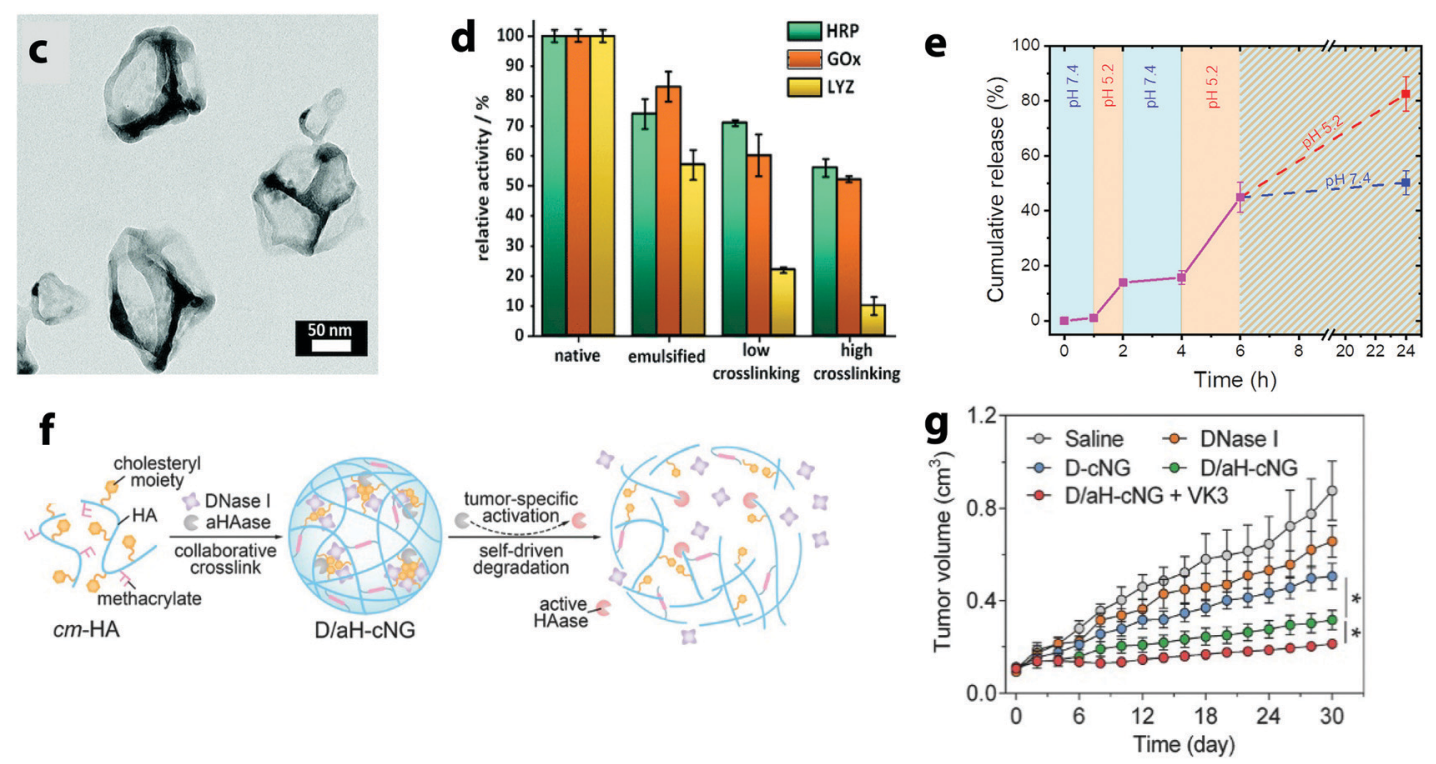

Fig. 6 (a) Schematic representation of nanoparticles/nanogels and nanocapsules, (b) scheme of the nanocapsule formation through interfacial polymerization in miniemulsion droplets, ${ }^{25}$ (c) TEM image of horseradish peroxidase nanocapsules, ${ }^{71}$ (d) relative enzymatic activity of horseradish peroxidase, glucose oxidase and lysozyme after emulsification and crosslinking as nanocapsules, ${ }^{71}$ (e) stop-and-go release of FITC-albumin from dextran nanogels by the variation of the $\mathrm{pH}$ value of the environment between 5.2 and $7.4{ }^{70}$ (f) schematic of self-assembly and tumor-specific self-degradation of the collaboratively crosslinked, hyaluronidase (HAase)-degradable hyaluronic acid nanogels (D/aH-cNG), (g) tumor size variation of the tumor-bearing nude mice treated with different formulations: saline control, DNase I, DNase I loaded nanogels (D-cNG), nanogels loaded with DNase I and the inactive aHAase concurrently ( $\mathrm{D} / \mathrm{aH}-\mathrm{cNG}$ ) and $\mathrm{D} / \mathrm{aH}$-cNG nanogels with the addition of vitamin $\mathrm{K} 3$ (VK3). ${ }^{72}$ (b) Reprinted with permission from ref. 25 . Copyright 2019 Wiley; (c and d) reprinted with permission from ref. 71. Copyright 2021 The Royal Chemical Society; (e) reprinted with permission from ref. 70. Copyright 2021 The American Chemical Society; (f and g) reprinted with permission from ref. 72. Copyright 2018 Wiley.

When the reaction kinetic is fast enough and the surface tension of the crosslinked polymer controlled, a solid polymer shell can be formed covering the precursor droplet. ${ }^{158}$ Many chemical reactions can occur at the droplet interface resulting in crosslinked core/shell structures. ${ }^{159}$ Various drugs can be loaded within the aqueous droplets, which usually results in very high encapsulation efficiencies. ${ }^{150,160}$ When mild reaction conditions are applied and the use of reagents and catalysts for the nanocapsule formation is controlled, sensitive BTAs can preserve their activity throughout this encapsulation process. 
For instance, such an approach allowed for the encapsulation of multiple enzymes in one nanocapsule with the aim of performing enzymatic cascade reactions. ${ }^{71}$ Similar strategies have been used to prepare both polymer and silica nanocapsules. ${ }^{71,161}$ All of the enzymes involved maintained their enzymatic activity after the encapsulation while they were successfully protected against proteases and heat. This strategy, of encapsulating biomacromolecular payload dissolved in the aqueous phase of an inverse miniemulsion by the formation of a shell at the droplet interface, has been used successfully to encapsulate enzyme and SiRNA in polymer and silica nanocapsules. ${ }^{162}$

Another technique yielding nanoparticles and nanogels without the aid of surfactants and organic solvents is precipitation polymerization. This process requires the use of a solvent, which can dissolve both the monomer and initiator or already preformed polymers and their crosslinker. Upon the initiation of the polymerization or crosslinking reaction, the polymer network becomes insoluble and precipitates as solid particles. ${ }^{71,157,163,164}$

Examples of application. The distinct structures produced by the different methods display different properties. Polymer nanocapsules are hollow containers in which a large variety of substances can be placed within the core reservoir. The resulting hosting capacity enables the delivery and the controlled release of a large variety of drugs from such DDSs. ${ }^{25}$ In comparison to solid nanoparticles, nanocapsules with a liquid core allow for the encapsulation of a much larger amount of payloads. ${ }^{165}$ For instance, poly(D,L lactide-co-caprolactone) nanocapsules were developed for the delivery of lysozyme and the therapeutic enzyme DNAse. ${ }^{166}$ By optimizing the monomer ratio of the copolymers employed, the lysozyme encapsulation efficiency reached $80 \%$. Further, by co-loading, a protein stabilizing sugar in the nanocapsules core, $98 \%$ of the lysozyme biological activity was preserved. Furthermore, by employing the identical nanocapsules formulation, 95\% of the biological activity of encapsulated DNAse was retained. In another approach, nanocapsules were prepared from the biomacromolecular therapeutic agent itself. $^{71}$ For instance, proteins and enzymes were crosslinked in an inverse miniemulsion to obtain solid protein nanocapsules with a retained biological activity of the biomacromolecules (Fig. 6c and d). The nanocapsules were synthesized by using horseradish peroxidase, glucose oxidase, and lysozyme as the main component of the nanocapsules, while the interior of the capsules allowed for the encapsulation of additional substances. Under a predetermined trigger, the enzyme nanoshell catalyzed the conversion of encapsulated material in situ, and the capsule acted as a catalytic nanoreactor. With this approach, the efficient mass fraction of active enzyme was increased by 50-1000 folds in comparison to conventional nanoreactors, where the enzyme is usually just encapsulated and not the building block of the nanosystem.

Nanogels, usually made of hydrogel, are polymer matrices with the ability to absorb a high amount of water in their network. Such a dynamic network of swollen hydrophilic polymers can contain ions, salts, polysaccharides or other stabilizing molecules co-dissolved with the drug and provides an optimal environment during the delivery process. Conversely, nanoparticles are built with an unswollen network of rigid crosslinked polymers and usually display a very high stability during the delivery process. In all cases, the release of biological agents incorporated in those polymer systems can occur by diffusion and mass transport through the polymer network. However, the release process can also be triggered by either the degradation of the nanoparticle or by a change in the structure of the polymer nanosystems, which occurs as a consequence of environmental signals present at the intended release site. ${ }^{167}$ In either case, the crosslinking density of the polymer network decreases while the mesh size is increased. The mesh size controls steric interactions between the drugs and the polymer network. When the mesh is larger than the drug, the drug release process is dominated by the diffusion of the drug through the polymer network. By controlling the mesh size, molecules of different sizes can be released with great control. For example, the release of fluorescently labeled albumin from ketone- and aldehyde-functionalized dextran-based nanogels was controlled by a reversible crosslinking of the polymer network. ${ }^{70}$ The hydrazone network density resulting from the reaction with an adipic acid dihydrazide crosslinker was tuned by the $\mathrm{pH}$-responsive equilibrium between hydrazide and hydrazone groups. Such dynamic covalent bonds undergo a reversible disassembly under an acidic $\mathrm{pH}$ value leading to the swelling of the nanogels by $c a$. $30 \%$. As the effective crosslinking density decreases, the swelling is triggered. It is fully reversible and can be used to induce a stop-and-go release of proteins from the nanogels (Fig. 6e).

In another example, a tumor-specific self-degradable nanogel composed of a hyaluronic acid network entrapping an acidactivatable hyaluronidase enzyme for the systemic delivery of anticancer proteins was developed. ${ }^{168}$ The hyaluronidase was modified with citraconic anhydride, which shielded the enzymatic activity. It can be reversibly activated as induced by the hydrolysis of the citraconic amide under acidic $\mathrm{pH}$ value. The mild acidity of the tumor microenvironment hence activates the hyaluronidase enzyme resulting in the swelling of the nanogel and triggering the release of the enzyme. The released enzyme is capable of degrading the nanogel network and further induces the release of the encapsulated anticancer protein, deoxyribonuclease I intracellularly (Fig. 6f). Further, the deoxyribonuclease I digested the DNA in the cell leading to the tumor cell death and an enhanced antitumor efficacy (Fig. 6g).

Some examples of polymer nanoparticles and nanogels used for the delivery of BTAs have proceeded to preclinical trials. For instance, PLGA nanoparticles loaded with the enzyme iduronate 2 -sulfatase were developed to treat the brain disease mucopolysaccharidosis type II by enzyme replacement therapy. ${ }^{74}$ This in vivo study, of a duration of six weeks, was performed on mice with mucopolysaccharidosis type II, showed a reduction of glycosaminoglycans typically accumulated in liver and brain tissues and an overall improvement of the brain pathology induced by the successful enzyme delivery after weekly administration of the nanoparticles. Another very recent study reported on the evaluation of cationic cholesteryl pullulan nanogels delivering the antigen protein pneumococcal 
surface protein A as a vaccine against Streptococcus pneumoniae bacteria, which can lead to respiratory infections. ${ }^{73}$ This study evaluated the immunogenicity and therapeutic efficacy of the nanogel vaccine in non-human primates (macaques). The results demonstrated that the vaccination of macaques with the nanogels induced high protection against pneumococcal infections. The macaques showed protection against pneumococcal intratracheal challenge by two mechanisms, the inhibition of possible lung inflammation and a decreased number of bacteria in the lungs.

Advantages and limitations. The encapsulation of BTAs in nanoparticles, nanogels or nanocapsules offers many advantages. The polymer network can be finely tuned to interrupt biomacromolecular drugs without their chemical modifications and thus preserve a high degree of their specific threedimensional structures. The crosslinking points in the polymer nanosystems endow those DDSs with great stability and allow for further surface functionalizations without the risk of premature drug release or the loss of physical integrity of the drug delivery system. ${ }^{169}$ The release of encapsulated BTAs can be controlled either by the degradation of the polymer itself or the swelling/deswelling of the polymer matrix. Moreover, the versatility of the preparation techniques provides the opportunity to incorporate an array of responsive units and smart properties either within the polymers or in the crosslinking points. One major challenge for the use of this type of DDSs is the encapsulation of active payload. There are two main strategies to get the BTAs inside the DDSs. The biomacromolecular therapeutic agent can be loaded after the synthesis of the nanoparticle, nanocapsule or nanogel. In this case, the low diffusion coefficient of those large macromolecules limits their efficient inclusion inside the DDSs. Alternatively, high encapsulation efficiency can be achieved by the formation of the DDSs around the BTAs, but in this instance, some of the harsh conditions associated with the formulation process, such as sonication, the use of organic solvents, or reaction condition like heat and UV irradiation, can disrupt the sensitive structure of the biomolecules and reduce the efficacy of the payload. ${ }^{166}$ Furthermore, the chemistries employed during the formation of the DDSs may lead to side reactions with the sensitive cargo. Hence, the development of new bioorthogonal reactions should be implemented more broadly to limit the occurrence of side reactions involving the therapeutic agent. Additionally, because of their size and limited diffusion, once a biomacromolecular therapeutic agent has been successfully encapsulated, it is not unusual for its release to be challenging, either slow or difficult to control. The crosslinking density employed in such systems is a critical aspect controlling the release of encapsulated macromolecules. For example, during the encapsulation of macromolecular payloads in polymer nanocapsules, a high crosslinking density of the polymer shell was shown to prevent its release from the DDS, ${ }^{170}$ while lower crosslinking may negatively influence the encapsulation efficiency. Hence, the crosslinking of the networks building the nanoparticles, nanogels or nanocapsules used as DDSs needs to be precisely tailored to the biomacromolecular therapeutic agent to be delivered to ensure the encapsulation while allowing for its successful release. Furthermore, especially in the case of nanoparticles and nanogels, the ratio of polymer used as the excipient compared to the amount of the therapeutic agent present in the system is often very large, with a typical nanoparticle composed of at least 10 times more excipient than active molecules. This can lead to undesired side effects emerging from the polymer material, such as the generation of immunogenic reaction against the polymer compounds, which compose most of the carrier. ${ }^{171}$

\section{Administration routes and toxicological evaluation of polymer drug delivery systems}

Polymer-based drug delivery systems provide many advantages, including increased stability of the formulations and versatility in terms of biomacromolecular therapeutic agents or their combinations to be delivered, compared to alternative delivery systems. However, for their efficient transfer from the lab to the bedside, we need to evaluate their efficacy, using different potential administration routes and their resulting toxicity after administration. The efficacy and risks associated with polymerbased DDSs are influenced by both their chemical compositions and the method chosen for their administration. As discussed in the previous sections, the choice of the ideal polymer formulation is highly influenced by the nature of the biomacromolecular therapeutic agent used. The choice of the administration route is usually a function of both the therapeutic agent used and the targeted site of action.

\section{Administration routes}

Potential administration routes for polymer DDSs include intravenous injections, oral administration, pulmonary inhalation, and transdermal delivery. ${ }^{172}$ Typically, the intravenous administration is accompanied by a rapid onset of action and high availability of the drug even with low doses. ${ }^{172}$ At a length scale of less than $200 \mathrm{~nm}$, polymer DDSs with encapsulated BTAs can be injected intravenously without any concern about embolization and blockages of the blood vessels. ${ }^{173}$ After the injection, they are able to permeate and move through the different organs and tissues. Depending on their surface chemistry, they can bind to cell surface receptors and enter target cells for intracellular delivery of the cargos. However, there are some risks associated with intravenous injection administration because of the direct exposure of the DDSs in the systemic circulation. The challenge is to maintain the DDSs in circulation in the blood for a long enough duration to ensure that the DDSs reach the site of action. ${ }^{174}$ In general, immediately after the injection, the DDSs suffer from a passive accumulation in the liver and the kidney. Those organs have the natural function to remove foreign material, such as viruses, bacteria or the nanoparticles used as DDSs from the bloodstream. ${ }^{175}$ After their injections, many DDSs are subjected to a nonspecific uptake by the mononuclear phagocyte system, composed of 
different phagocytic cells, such as resident macrophages commonly found in the spleen, lymph nodes, and the liver. ${ }^{176}$ Both undesired effects depend on the size and surface functionalization of the DDSs and may be prevented by adjusting those properties. $^{177}$

Oral delivery is one of the administration routes associated with the largest patient compliance. Many biodegradable and non-biodegradable polymeric DDSs have been used for oral drug delivery. For instance, a study of PLGA nanoparticles encapsulating curcumin, a water-insoluble drug, analyzed the pharmacokinetics and bioavailability of the formulation in vivo and showed a 6-fold higher oral bioavailability of the nanoparticulate drug delivery system compared with the pure drug. ${ }^{178}$ In another example, polydopamine nanoparticles loaded with gambogenic acid, an anti-cancer drug, showed that after oral administration in mice, the polymer DDS increased the plasma drug concentration almost 3 times without apparent toxicity to the major organs. ${ }^{179}$ One of the major challenges associated with oral delivery is the transit through the gastrointestinal tract and the very acidic $\mathrm{pH}$ of this environment, which also contain a complex enzymatic system. Protecting the sensitive structure of the biomacromolecular therapeutic agent, such as genetic material, under such harsh conditions is difficult. Even ensuring the stability of the DDSs is a challenging task, even more so for systems like polyplexes and LBL assemblies relying on $\mathrm{pH}$ and ionic strength to prevent their dissociation. Consequently, they can be destabilized by the environmental cues encountered in the gastrointestinal tract. Different strategies have been implemented to improve the stability of the DDSs. For example, to increase the stability of a polyplex used for the treatment of diabetes by oral administration used the presence of an additive to stabilize the DDSs. ${ }^{180}$ In this system, a plasmid DNA was complexed with protamine, but the protamine used was functionalized with taurocholic acid. The resulting nonviral oral gene delivery polyplex protected the integrity of the plasmid in gastric juice and prevented its degradation by proteolytic enzymes in the environment. Furthermore, following administration, the results show significant gene expression in in vitro, in vivo, and ex vivo experiments. After administering a single oral dose of the formulation, normal blood glucose levels were maintained for up to 7 days in three diabetic mouse models and for 14 days in monkeys with diabetic conditions. Those impressive results were attributed to the incorporation of taurocholic acid into the delivery system, which led to an improvement in the stability of the DDS in a broad range of $\mathrm{pH}$, preserving the integrity of the polyplex.

Pulmonary delivery also has some advantages since lungs have a high solute permeability, a large surface area for absorption, and that pulmonary delivery is non-invasive. ${ }^{181}$ For their use in pulmonary delivery, the DDSs should have the ability to be transferred into an aerosol, for example, by the nebulization of a colloidal suspension. ${ }^{182}$ Then, after the inhalation, the DDSs loaded with the BTA may provide a sustained release of the drug in the lung tissue, minimizing the required dosage frequency. ${ }^{181}$ For instance, insulin-loaded nanoparticles composed of poly(butyl cyanoacrylate) extended the blood glucose level reduction over $20 \mathrm{~h}$ compared to the nonencapsulated drug after pulmonary administration to rats. ${ }^{183}$ Furthermore, the local pulmonary administration of DDSs may reduce potential side effects on other organs. An interesting example showed the successful pulmonary administration of PEI-siRNA polyplexes using a liquid aerosol device. In tumorbearing mice models, the local delivery of the siRNA polyplexes resulted in a significant accumulation of the siRNA in the lungs leading to an inhibition of the tumor growth of the metastatic lung cancer. ${ }^{182}$

Transdermal drug delivery is another possibility for a noninvasive systemic drug administration, but the inherent protective function of the skin often leads to low permeability toward external substances. ${ }^{184}$ In general, the molecular weight of the drug is a significant limitation for the dermal delivery of therapeutics, and drugs with a molecular weight of more than 500 Da cannot penetrate through the skin passively. ${ }^{185}$ However, the formulation of a drug or BTA with the appropriate polymer DDSs can increase the transdermal delivery. The penetration ability of the DDSs is affected mainly by their size. ${ }^{185}$ For instance, penetration experiments in porcine skin revealed that polystyrene nanoparticles of $20 \mathrm{~nm}$ size permeate more efficiently in the skin than bigger ones $(200 \mathrm{~nm}) .{ }^{186}$ Furthermore, polymer DDSs are also able to penetrate skin follicles and hence allow for the opportunity of directed intrafollicular drug release. ${ }^{187}$ This release can be initiated by extrinsic or intrinsic triggers, such as low power density UVA radiation. For example, ultraviolet A-responsive polyurethane nanocapsules showed sufficient follicular penetration and intrafollicular drug release on an ex vivo porcine ear skin model while showing excellent biocompatibility before and after UVAinduced cleavage. ${ }^{187}$

\section{Toxicology of polymer DDSs}

The use of polymer-based DDSs for the delivery of biomacromolecular payloads suffers from the same limitations as DDSs used to deliver smaller therapeutic agents. ${ }^{188}$ In addition to the toxicity of the DDSs themselves, the toxicity of the polymers used and their degradation products need to be taken into account, as well as the toxicity potentially associated with an off-target delivery of the payload. The clinical application of polymer DDSs needs to be critically controlled, and the inherent toxicity of all components of the formulation has to be screened. ${ }^{189,190}$ However, there is a pervasive challenge in the study of polymer nanosystems used as DDSs; it is critical to understand and compare results obtained with different systems, there could be large variations in how such studies are performed and how the results are reported. This lack of standardization impede the community to draw clear conclusions on the interactions and process occurring at such bio-nano interface. $^{191}$

Several factors affect the toxicity of polymer DDSs. On the one hand, those include colloidal properties, such as the size and shape of the DDSs, the surface charge, and the surface functionalization. The size is one of the most critical parameters in influencing the toxicity of the DDS. Typically, the 
smaller the size of the nano-DDSs, the higher the associated toxicity will be. This phenomenon can be attributed to the increasing ability of smaller nanoparticles to cross biological barriers and reach different organs without being filtered by the liver or spleen. ${ }^{192}$ Inversely, if the DDSs are too large, they will be rapidly filtered out and cause local toxicity to the liver and spleen. Furthermore, the surface charge of the DDSs also significantly influences the interaction between the nanocarriers and the body. In general, nanocarriers with positive charges show larger toxicity compared to DDSs with negative surface charges. ${ }^{193}$

Other parameters affecting the toxicity of the DDSs are related to the properties of the polymer material. These include the origin of the polymer, the molecular weight, the hydrophilicity, the charge, and the biodegradability. Polymers from natural sources are usually more biocompatible and biodegradable compared to some synthetic polymers. Their degradation products are mostly non-toxic, even in high concentrations, since they are endogenous. Such systems made of, for example, hyaluronic acid, chitosan, starch or dextran, have been extensively used as non-toxic building blocks for the preparation of DDSs. For instance, the toxicity of chitosan DDSs upon repeated oral administrations was studied extensively. A large variety of chitosan nanosystems, such as nanoparticles composed of chitosan/alginate, chitosan/glutamic acid or oleoyl-carboxy methyl chitosan, as well as chitosan-coated PLGA DDSs, have been studied in different animal models, such rats, mice, and carps, all those studies revealed no or very limited in vivo toxicity. ${ }^{194-198}$

Many synthetic biodegradable polymer building blocks employed in the design of drug delivery systems, such as poly(lactic acid), poly(glycolic acid), and poly(lactide-co-glycolide), have already been approved by the US Food and Drug Administration and the European Medicines Agency due to their safety, biocompatibility, and successful biodegradation during in vitro and in vivo studies. ${ }^{190}$ Hence, the design of nanoparticulate formulations using such polymers shows a high potential for a satisfactory toxicological response. For instance, PLGA nanoparticles used in A549 human lung cancer cells at a large concentration range $\left(300-5000 \mu \mathrm{g} \mathrm{mL}{ }^{-1}\right)$ showed no toxicity even at the highest concentration. ${ }^{199}$ Similarly, the administration of poly( $n$-butylcyano-acrylate) nanoparticles coated with polysorbate 80 in vivo in rats showed almost no cytotoxic or inflammatory effects at therapeutic concentrations lower than $500 \mu \mathrm{g} \mathrm{mL}^{-1}{ }^{200}$ Additionally, the use of biological coating materials such as chitosan or serum proteins may even further increase the biocompatibility of polymer nanostructures. In this regard, an interesting study demonstrated a reduction of the cytotoxicity of PLGA nanoparticles stabilized with bovine serum protein when compared to synthetic coating materials in cultured lung cancer cells. ${ }^{201}$

At the opposite end of the spectra, charged polymers like those used to prepare delivery carriers based on electrostatic interaction, such as polyplexes or LbL assemblies, may exhibit critical toxicological properties. Cationic polymers are known to interact with the anionic molecules present at the surface of different types of cells, and the use of such polymers can damage cells and tissues. ${ }^{202}$ For instance, the toxicology of poly(ethyleneimine) was extensively studied and the results revealed two distinct stages of the cytotoxicity of PEI. ${ }^{203}$ The first stage of cytotoxicity is a rapid response; for example, PEI/pDNA polyplexes and free PEI polymers bound to cell membranes induced membrane damages in three clinically relevant human cell lines (Jurkat $\mathrm{T}$ cells, umbilical vein endothelial cells, and THLE3 hepatocyte-like cells) within 30 min following the exposure. ${ }^{203}$ Then, the second stage of cytotoxicity occurs after longer exposition to the DDSs; for the same PEI polyplex, severe apoptotic changes of the cells were observed after $24 \mathrm{~h}$ of exposure. Hence, to avoid the potential cytotoxicity, cationic polymers used in drug delivery systems could be designed with the inclusion of degradable functionalities. Some promising examples of this approach include the crosslinking of low-molecular-weight PEI with degradable linkers, such as ester bonds, disulfide bonds, and ketal functions. ${ }^{204,205}$ Anther possible approach is the coating of cationic polymer DDSs with neutral or anionic polymers in order to increase their biocompatibility. For instance, cationic polymer micelles composed of a diblock amphiphilic copolymer of PEI and poly(DL-lactic acid) were coated with polycarboxylic acid dextran after the binding of survivin-shRNA. ${ }^{206}$ The polymer micelles were used in anti-cancer therapy and resulted in significant inhibition of the tumor growth in C26 tumor-bearing mice without a sign of systemic toxicity as monitored by the bodyweight loss and survival rate of the mice during the treatment.

\section{Conclusion}

Biomacromolecular therapeutic agents are promising candidates for the treatment of many complex and severe diseases. However, due to their inherent low stability and poor delivery potential under in vivo conditions, the design of tailored and multi-functional DDSs is essential. The ideal drug delivery system for BTAs should overcome the following specific challenges: preserve and protect the vulnerable structure of the biomacromolecule, and enable the intracellular uptake of the therapeutic agent. Traditional DDSs, such as liposomal formulations, have shown great success in the delivery of BTAs. However, these DDSs are inherently unable to simultaneously solve all the challenges encountered in the delivery BTAs.

Currently, because of advances in protein and oligonucleotide synthesis and isolation, the development and approval of new biomacromolecular therapeutic agents are increasing. Consequently, the design of appropriate delivery systems for such drugs remains a significant challenge to tackle. While polymer-conjugates of several biomacromolecular therapeutic agents have already been approved and are currently being used by patients, other types of polymer DDSs have primarily only been evaluated in in vitro and in vivo screenings, with a very small number of cases proceeding to preclinical and clinical trials. This situation can be mainly attributed to the complexity in their design, which can become a hurdle when it 
comes time to scale up the production while maintaining control over the chemistry, structure, and properties during this manufacturing step. The apparent dilemma in the field is that successful DDSs require a sophisticated structure and smart functionalities to simultaneously protect the biomacromolecular therapeutic agent, successfully deliver the payload to the intended site of action, and controlled the release, but such complex systems are challenging to scale up. Therefore, it is likely that DDSs combining the desired pharmacokinetic properties with some degree of design simplicity will more easily translate from the lab to applications. To allow for more complex DDSs to thrive and get approval by the authorities for human use, more attention has to be paid to the development of approaches with a high batch-to-batch reproducibility compatible with industrial scale-up production. ${ }^{207}$

The success rate of new treatments entering clinical trials is low no matter the type of drugs or delivery systems. ${ }^{208}$ However, this is particularly the case for nanodelivery systems where the translational efficiency and the clinical success rate are both (still) very low. ${ }^{22}$ In order to change this situation, a more systematic study of the nano-bio interaction is needed, ${ }^{191}$ and in addition to some degree of standardization in the field, a broader scope of models both in terms of diseases and animal models should be considered. ${ }^{209}$

Polymer drug delivery systems are unique among DDSs, since they can combine multiple functionalities in one nanosystem due to the flexibility in their synthesis. The resulting polymer DDSs can address all the challenges faced by the delivery of biomacromolecules. Their unique strength lies in the unlimited variety of potential starting materials resulting in precisely controlled and rationally designed systems. The different architectures of polymer DDSs provide versatile strategies for the successful delivery of the most challenging payloads.

Some key advantages of polymer DDSs are their high stability in complex environments even at low concentration, the ability to introduce active targeting ligands but also to tune passive targeting by controlling their size and shape, their high loading efficiency, their ability to co-deliver multiple therapeutics regardless of their hydrophilicity and molecular weight, and the selective and controlled release of cargo.

\section{Conflicts of interest}

There are no conflicts to declare.

\section{Acknowledgements}

The authors thank the Else Kröner-Fresenius-Stiftung, the Max Planck Society, the MaxSynBio consortium, and the Deutsche Forschungsgemeinschaft (SFB1066) for their financial support. The help of Stefan Schuhmacher with graphical design is acknowledged. Open Access funding provided by the Max Planck Society.

\section{Notes and references}

1 S. Mitragotri, P. A. Burke and R. Langer, Nat. Rev. Drug Discovery, 2014, 13, 655-672.

2 M. Muttenthaler, G. F. King, D. J. Adams and P. F. Alewood, Nat. Rev. Drug Discovery, 2021, 20, 309-325.

3 J. A. Kulkarni, D. Witzigmann, S. B. Thomson, S. Chen, B. R. Leavitt, P. R. Cullis and R. van der Meel, Nat. Nanotechnol., 2021, 16, 630-643.

4 B. C. Evans, R. B. Fletcher, K. V. Kilchrist, E. A. Dailing, A. J. Mukalel, J. M. Colazo, M. Oliver, J. Cheung-Flynn, C. M. Brophy, J. W. Tierney, J. S. Isenberg, K. D. Hankenson, K. Ghimire, C. Lander, C. A. Gersbach and C. L. Duvall, Nat. Commun., 2019, 10, 1-19.

5 G. Walsh, Appl. Microbiol. Biotechnol., 2005, 67, 151-159.

6 C. Sheridan, Nat. Rev. Drug Discovery, 2006, 5, 445.

7 A. L. Nelson, E. Dhimolea and J. M. Reichert, Nat. Rev. Drug Discovery, 2010, 9, 767-774.

8 G. Walsh, Nat. Biotechnol., 2018, 36, 1136-1145.

9 A. Wolfram Julie and J. K. Donahue, J. Am. Heart Assoc., 2013, 2, 119.

10 I. Lostalé-Seijo and J. Montenegro, Nat. Rev. Chem., 2018, 2, 258-277.

11 N. G. Rainov, Hum. Gene Ther., 2000, 11, 2389-2401.

12 W. J. Marks, J. L. Ostrem, L. Verhagen, P. A. Starr, P. S. Larson and R. A. Bakay, Lancet Neurol., 2008, 7, 400-408.

13 R. T. Bartus, T. L. Baumann, J. Siffert, C. D. Herzog, R. Alterman, N. Boulis, D. A. Turner, M. Stacy, A. E. Lang, A. M. Lozano and C. W. Olanow, Neurology, 2013, 80, 1698.

14 F. P. Polack, S. J. Thomas, N. Kitchin, J. Absalon, A. Gurtman, S. Lockhart, J. L. Perez, G. Pérez Marc, E. D. Moreira, C. Zerbini, R. Bailey, K. A. Swanson, S. Roychoudhury, K. Koury, P. Li, W. V. Kalina, D. Cooper, R. W. Frenck, L. L. Hammitt, Ö. Türeci, H. Nell, A. Schaefer, S. Ünal, D. B. Tresnan, S. Mather, P. R. Dormitzer, U. Şahin, K. U. Jansen and W. C. Gruber, N. Engl. J. Med., 2020, 383, 2603-2615.

15 J. Houseley and D. Tollervey, Cell, 2009, 136, 763-776.

16 T. Kisby, A. Yilmazer and K. Kostarelos, Nat. Nanotechnol., 2021, 16, 843-850.

17 D. J. A. Crommelin, T. J. Anchordoquy, D. B. Volkin, W. Jiskoot and E. Mastrobattista, J. Pharm. Sci., 2021, 110, 997-1001.

18 F. Vahedifard and K. Chakravarthy, Emergent Mater., 2021, 4, 75-99.

19 A. C. Anselmo and S. Mitragotri, Bioeng. Trans. Med., 2019, 4, e10143.

20 O. S. Fenton, K. N. Olafson, P. S. Pillai, M. J. Mitchell and R. Langer, Adv. Mater., 2018, 30, 1705328.

21 D. Bobo, K. J. Robinson, J. Islam, K. J. Thurecht and S. R. Corrie, Pharm. Res., 2016, 33, 2373-2387.

22 H. Zhong, G. Chan, Y. Hu, H. Hu and D. Ouyang, Pharmaceutics, 2018, 10, 263.

23 K. R. Sims, J. P. Maceren, A. I. Strand, B. He, C. Overby and D. S. W. Benoit, RSC Adv., 2020, 10, 2513-2518. 
24 Z. Tang, C. He, H. Tian, J. Ding, B. S. Hsiao, B. Chu and X. Chen, Prog. Polym. Sci., 2016, 60, 86-128.

25 B. Iyisan and K. Landfester, Macromol. Rapid Commun., 2019, 40, 1800577.

26 N. Kamaly, B. Yameen, J. Wu and O. C. Farokhzad, Chem. Rev., 2016, 116, 2602-2663.

27 J. Wang, Y. Li and G. Nie, Nat. Rev. Mater., 2021, 6, 766-783.

28 L. Zhao, T.-h. Ren and D. D. Wang, Acta Pharmacol. Sin., 2012, 33, 1339-1347.

29 K. A. Dill, Biochemistry, 1990, 29, 7133-7155.

30 S. Burge, G. N. Parkinson, P. Hazel, A. K. Todd and S. Neidle, Nucleic Acids Res., 2006, 34, 5402-5415.

31 S. Jung and I. Kwon, Polym. Chem., 2016, 7, 4584-4598.

32 K. Samejima and W. C. Earnshaw, Nat. Rev. Mol. Cell Biol., 2005, 6, 677-688.

33 C. López-Otín and J. S. Bond, J. Biol. Chem., 2008, 283, 30433-30437.

34 M. Werle and A. Bernkop-Schnürch, Amino Acids, 2006, 30, 351-367.

35 G. B. Bolli, D. Kerr, R. Thomas, E. Torlone, A. SolaGazagnes, E. Vitacolonna, J. L. Selam and P. D. Home, Diabetes Care, 2009, 32, 1170.

36 F. Scaletti, J. Hardie, Y.-W. Lee, D. C. Luther, M. Ray and V. M. Rotello, Chem. Soc. Rev., 2018, 47, 3421-3432.

37 J. Lombard, Biol. Direct, 2014, 9, 32.

38 P. Matsson and J. Kihlberg, J. Med. Chem., 2017, 60, 1662-1664.

39 N. J. Yang and M. J. Hinner, in Site-Specific Protein Labeling: Methods and Protocols, ed. A. Gautier and M. J. Hinner, Springer, New York, 2015.

40 F.-S. Du, Y. Wang, R. Zhang and Z.-C. Li, Soft Matter, 2010, 6, 835-848.

41 G. Gregoriadis, N. Engl. J. Med., 1976, 295, 765-770.

42 Nat. Rev. Mater., 2021, 6, 99.

43 J. A. Zuris, D. B. Thompson, Y. Shu, J. P. Guilinger, J. L. Bessen, J. H. Hu, M. L. Maeder, J. K. Joung, Z.-Y. Chen and D. R. Liu, Nat. Biotechnol., 2015, 33, 73-80.

44 R. Münter, K. Kristensen, D. Pedersbæk, J. B. Larsen, J. B. Simonsen and T. L. Andresen, Nanoscale, 2018, 10, 22720-22724.

45 D. J. McClements, Adv. Colloid Interface Sci., 2018, 253, $1-22$.

46 S. H. Lee, Y. Y. Kang, H.-E. Jang and H. Mok, Adv. Drug Deliv. Rev., 2016, 104, 78-92.

47 I. Ekladious, Y. L. Colson and M. W. Grinstaff, Nat. Rev. Drug Discovery, 2019, 18, 273-294.

48 Y. Wu, D. Y. W. Ng, S. L. Kuan and T. Weil, Biomater. Sci., 2015, 3, 214-230.

49 E. Steiert, J. Ewald, A. Wagner, U. A. Hellmich, H. Frey and P. R. Wich, Polym. Chem., 2020, 11, 551-559.

50 I. Cobo, M. Li, B. S. Sumerlin and S. Perrier, Nat. Mater., 2015, 14, 143-159.

51 J. D. Schulz, M. Patt, S. Basler, H. Kries, D. Hilvert, M. A. Gauthier and J.-C. Leroux, Adv. Mater., 2016, 28, 1455-1460.

52 S. Averick, O. Karácsony, J. Mohin, X. Yong, N. M. Moellers, B. F. Woodman, W. Zhu, R. A. Mehl, A. C. Balazs,
T. Kowalewski and K. Matyjaszewski, Angew. Chem., Int. Ed., 2014, 53, 8050-8055.

53 H. Cho, T. Daniel, Y. J. Buechler, D. C. Litzinger, Z. Maio, A.-M. H. Putnam, V. S. Kraynov, B.-C. Sim, S. Bussell, T. Javahishvili, S. Kaphle, G. Viramontes, M. Ong, S. Chu, B. Gc, R. Lieu, N. Knudsen, P. Castiglioni, T. C. Norman, D. W. Axelrod, A. R. Hoffman, P. G. Schultz, R. D. DiMarchi and B. E. Kimmel, Proc. Natl. Acad. Sci. U. S. A., 2011, 108, 9060.

54 A. C. Braun, M. Gutmann, T. D. Mueller, T. Lühmann and L. Meinel, J. Controlled Release, 2018, 279, 17-28.

55 C. Bao, J. Chen, D. Li, A. Zhang and Q. Zhang, Polym. Chem., 2020, 11, 1386-1392.

56 Y.-A. Heo, Y. Y. Syed and S. J. Keam, Drugs, 2019, 79, 767-777.

57 T. M. Herndon, S. G. Demko, X. Jiang, K. He, J. E. Gootenberg, M. H. Cohen, P. Keegan and R. Pazdur, The Oncologist, 2012, 17, 1323-1328.

58 A. M. M. Eggermont, S. Suciu, M. Santinami, A. Testori, W. H. J. Kruit, J. Marsden, C. J. A. Punt, F. Salès, M. Gore, R. MacKie, Z. Kusic, R. Dummer, A. Hauschild, E. Musat, A. Spatz and U. Keilholz, The Lancet, 2008, 372, 117-126.

59 K. Kim, H. S. Hwang, M. S. Shim, Y.-Y. Cho, J. Y. Lee, H. S. Lee and H. C. Kang, Colloids Surf., B, 2019, 184, 110497.

60 Y. Dong, T. Yu, L. Ding, E. Laurini, Y. Huang, M. Zhang, Y. Weng, S. Lin, P. Chen, D. Marson, Y. Jiang, S. Giorgio, S. Pricl, X. Liu, P. Rocchi and L. Peng, J. Am. Chem. Soc., 2018, 140, 16264-16274.

61 O. K. Appelbe, B.-K. Kim, N. Rymut, J. Wang, S. J. Kron and Y. Yeo, Cancer Gene Ther., 2018, 25, 196-206.

62 L. Buscail, B. Bournet, F. Vernejoul, G. Cambois, H. Lulka, N. Hanoun, M. Dufresne, A. Meulle, A. Vignolle-Vidoni, L. Ligat, N. Saint-Laurent, F. Pont, S. Dejean, M. Gayral, F. Martins, J. Torrisani, O. Barbey, F. Gross, R. Guimbaud, P. Otal, F. Lopez, G. Tiraby and P. Cordelier, Mol. Ther., 2015, 23, 779-789.

63 J. E. Zuckerman, I. Gritli, A. Tolcher, J. D. Heidel, D. Lim, R. Morgan, B. Chmielowski, A. Ribas, M. E. Davis and Y. Yen, Proc. Natl. Acad. Sci. U. S. A., 2014, 111, 11449.

64 B. G. De Geest, M. A. Willart, B. N. Lambrecht, C. Pollard, C. Vervaet, J. P. Remon, J. Grooten and S. De Koker, Angew. Chem., Int. Ed., 2012, 51, 3862-3866.

65 Z. J. Deng, S. W. Morton, E. Ben-Akiva, E. C. Dreaden, K. E. Shopsowitz and P. T. Hammond, ACS Nano, 2013, 7, 9571-9584.

66 F. Wang, J. Gao, J. Xiao and J. Du, Nano Lett., 2018, 18, 5562-5568.

67 Q. Ji, J. Hou, X. Yong, G. Gong, M. Muddassir, T. Tang, J. Xie, W. Fan and X. Chen, Adv. Mater., 2021, 33, 2007798.

68 C.-Y. Lin, F. Perche, M. Ikegami, S. Uchida, K. Kataoka and K. Itaka, J. Controlled Release, 2016, 235, 268-275.

69 J. H. Lam, A. K. Khan, T. A. Cornell, T. W. Chia, R. J. Dress, W. W. W. Yeow, N. K. Mohd-Ismail, S. Venkatraman, K. T. Ng, Y.-J. Tan, D. E. Anderson, F. Ginhoux and M. Nallani, ACS Nano, 2021, 15, 15754-15770. 
70 M. S. Alkanawati, M. Machtakova, K. Landfester and H. Thérien-Aubin, Biomacromolecules, 2021, 22, 2976-2984.

71 M. Machtakova, S. Han, Y. Yangazoglu, I. Lieberwirth, H. Thérien-Aubin and K. Landfester, Nanoscale, 2021, 13, 4051-4059.

72 Q. Zhu, X. Chen, X. Xu, Y. Zhang, C. Zhang and R. Mo, Adv. Funct. Mater., 2018, 28, 1707371.

73 R. Nakahashi-Ouchida, Y. Uchida, Y. Yuki, Y. Katakai, T. Yamanoue, H. Ogawa, Y. Munesue, N. Nakano, K. Hanari, T. Miyazaki, Y. Saito, S. Umemoto, S.-I. Sawada, R. Mukerji, D. E. Briles, Y. Yasutomi, K. Akiyoshi and H. Kiyono, Vaccine, 2021, 39, 3353-3364.

74 L. Rigon, M. Salvalaio, F. Pederzoli, E. Legnini, J. T. Duskey, F. D’Avanzo, C. De Filippis, B. Ruozi, O. Marin, M. A. Vandelli, I. Ottonelli, M. Scarpa, G. Tosi and R. Tomanin, Int. J. Mol. Sci., 2019, 20, 214.

75 H. Schlaad, Bio-synthetic Polymer Conjugates, SpringerVerlag, Berlin Heidelberg, 2013.

76 E. M. Pelegri-O'Day and H. D. Maynard, Acc. Chem. Res., 2016, 49, 1777-1785.

77 W. Zhao, F. Liu, Y. Chen, J. Bai and W. Gao, Polymer, 2015, 66, 1-10.

78 B. S. Lele, H. Murata, K. Matyjaszewski and A. J. Russell, Biomacromolecules, 2005, 6, 3380-3387.

79 K. M. Mansfield and H. D. Maynard, ACS Macro Lett., 2018, 7, 324-329.

80 A. Bastida, P. Sabuquillo, P. Armisen, R. FernándezLafuente, J. Huguet and J. M. Guisán, Biotechnol. Bioeng., 1998, 58, 486-493.

81 R. D. Schmid and R. Verger, Angew. Chem., Int. Ed., 1998, 37, 1608-1633.

82 J. Khandare and T. Minko, Prog. Polym. Sci., 2006, 31, 359-397.

83 D. Charych, S. Khalili, V. Dixit, P. Kirk, T. Chang, J. Langowski, W. Rubas, S. K. Doberstein, M. Eldon, U. Hoch and J. Zalevsky, PLoS One, 2017, 12, e0179431.

84 D. H. Charych, U. Hoch, J. L. Langowski, S. R. Lee, M. K. Addepalli, P. B. Kirk, D. Sheng, X. Liu, P. W. Sims, L. A. VanderVeen, C. F. Ali, T. K. Chang, M. Konakova, R. L. Pena, R. S. Kanhere, Y. M. Kirksey, C. Ji, Y. Wang, J. Huang, T. D. Sweeney, S. S. Kantak and S. K. Doberstein, Clin. Cancer Res., 2016, 22, 680.

85 H. Lv, S. Zhang, B. Wang, S. Cui and J. Yan, J. Controlled Release, 2006, 114, 100-109.

86 M. Lucius, R. Falatach, C. McGlone, K. Makaroff, A. Danielson, C. Williams, J. C. Nix, D. Konkolewicz, R. C. Page and J. A. Berberich, Biomacromolecules, 2016, 17, 1123-1134.

87 X. Pang, Y. Jiang, Q. Xiao, A. W. Leung, H. Hua and C. Xu, J. Controlled Release, 2016, 222, 116-129.

88 S. L. Turgeon, C. Schmitt and C. Sanchez, Curr. Opin. Colloid Interface Sci., 2007, 12, 166-178.

89 C. G. de Kruif, F. Weinbreck and R. de Vries, Curr. Opin. Colloid Interface Sci., 2004, 9, 340-349.

90 E. Kizilay, A. B. Kayitmazer and P. L. Dubin, Adv. Colloid Interface Sci., 2011, 167, 24-37.
91 C. H. Porcel and J. B. Schlenoff, Biomacromolecules, 2009, 10, 2968-2975.

92 Q. Wang and J. B. Schlenoff, Macromolecules, 2014, 47, 3108-3116.

93 R. G. Winkler and A. G. Cherstvy, in Polyelectrolyte Complexes in the Dispersed and Solid State I: Principles and Theory, ed. M. Müller, Springer, Berlin, 2014, pp. 1-56.

94 M. Ogris, S. Brunner, S. Schüller, R. Kircheis and E. Wagner, Gene Ther., 1999, 6, 595-605.

95 J. V. D. Gucht, E. Spruijt, M. Lemmers and M. A. Cohen Stuart, J. Colloid Interface Sci., 2011, 361, 407-422.

96 D. Priftis and M. Tirrell, Soft Matter, 2012, 8, 9396-9405.

97 E. Lai and J. H. van Zanten, Biophys. J., 2001, 80, 864-873.

98 A. Dinari, T. T. Moghadam, M. Abdollahi and M. Sadeghizadeh, Sci. Rep., 2018, 8, 8112.

99 F. Lotfipour, S. Hallaj-Nezhadi, H. Valizadeh, S. Dastmalchi, B. Baradaran, M. B. Jalali and F. Dobakhti, J. Pharm. Pharm. Sci., 2011, 14, 181-195.

100 A. K. Blakney, G. Yilmaz, P. F. McKay, C. R. Becer and R. J. Shattock, Biomacromolecules, 2018, 19, 2870-2879.

101 A. Ahmad, F. Khan, R. K. Mishra and R. Khan, J. Med. Chem., 2019, 62, 10475-10496.

102 K.-K. H. Kuo, P.-J. Hsiao, W.-T. Chang, S.-C. Chuang, Y.-H. Yang, K. Wuputra, C.-C. Ku, J.-B. Pan, C.-P. Li, K. Kato, C.-J. Liu, D.-C. Wu and K. K. Yokoyama, Cancers, 2021, 13, 3920.

103 ClinicalTrials.gov. National Library of Medicine (U.S.)., Effect of intratumoral injection of gene therapy for locally advanced pancreatic cancer (THERGAP-02), Identifier NCT02806687, Retrieved October 05, 2021 from https:// clinicaltrials.gov/ct2/show/study/NCT02806687.

104 W. R. Gombotz and D. K. Pettit, Bioconjugate Chem., 1995, 6, 332-351.

105 K. A. Mislick and J. D. Baldeschwieler, Proc. Natl. Acad. Sci. U. S. A., 1996, 93, 12349-12354.

106 A. Akinc, M. Thomas, A. M. Klibanov and R. Langer, J. Gene Med., 2005, 7, 657-663.

107 T. Bus, A. Traeger and U. S. Schubert, J. Mater. Chem. B, 2018, 6, 6904-6918.

108 E. C. Freeman, L. M. Weiland and W. S. Meng, J. Biomater. Sci., Polym. Ed., 2013, 24, 398-416.

109 H. Chang, J. Lv, X. Gao, X. Wang, H. Wang, H. Chen, X. He, L. Li and Y. Cheng, Nano Lett., 2017, 17, 1678-1684.

110 V. Postupalenko, D. Desplancq, I. Orlov, Y. Arntz, D. Spehner, Y. Mely, B. P. Klaholz, P. Schultz, E. Weiss and G. Zuber, Angew. Chem., Int. Ed., 2015, 54, 10583-10586.

111 J.-G. Piao, J.-J. Yan, M.-Z. Wang, D.-C. Wu and Y.-Z. You, Biomater. Sci., 2014, 2, 390-398.

112 X. Q. Liu and C. Picart, Adv. Mater., 2016, 28, 1295-1301.

113 P. T. Hammond, Adv. Mater., 2004, 16, 1271-1293.

114 J. J. Richardson, M. Björnmalm and F. Caruso, Science, 2015, 348, aaa2491.

115 J. J. Richardson, J. Cui, M. Björnmalm, J. A. Braunger, H. Ejima and F. Caruso, Chem. Rev., 2016, 116, 14828-14867.

116 A. M. T. San Juan, T. Rodgers, C. Bedolla, F. Noriega and G. Romero, J. Appl. Polym. Sci., 2020, 137, 49377. 
117 Y. Yan, M. Björnmalm and F. Caruso, Chem. Mater., 2014, 26, 452-460.

118 S. Zhao, F. Caruso, L. Dähne, G. Decher, B. G. D. Geest, J. Fan, N. Feliu, Y. Gogotsi, P. T. Hammond, M. C. Hersam, A. Khademhosseini, N. Kotov, S. Leporatti, Y. Li, F. Lisdat, L. M. Liz-Marzán, S. Moya, P. Mulvaney, A. L. Rogach, S. Roy, D. G. Shchukin, A. G. Skirtach, M. M. Stevens, G. B. Sukhorukov, P. S. Weiss, Z. Yue, D. Zhu and W. J. Parak, ACS Nano, 2019, 13, 6151-6169.

119 F. Caruso and C. Schüler, Langmuir, 2000, 16, 9595-9603.

120 A. Küchler, M. Yoshimoto, S. Luginbühl, F. Mavelli and P. Walde, Nat. Nanotechnol., 2016, 11, 409-420.

121 R. Yang, J. Xu, L. Xu, X. Sun, Q. Chen, Y. Zhao, R. Peng and Z. Liu, ACS Nano, 2018, 12, 5121-5129.

122 M. D. Shin, S. Shukla, Y. H. Chung, V. Beiss, S. K. Chan, O. A. Ortega-Rivera, D. M. Wirth, A. Chen, M. Sack, J. K. Pokorski and N. F. Steinmetz, Nat. Nanotechnol., 2020, 15, 646-655.

123 T. Tang, T. Weng, H. Jia, S. Luo, Y. Xu, L. Li and P. Zhang, Biomater. Sci., 2019, 7, 715-732.

124 F.-X. Xiao, M. Pagliaro, Y.-J. Xu and B. Liu, Chem. Soc. Rev., 2016, 45, 3088-3121.

125 A. M. Jhaveri and V. P. Torchilin, Front. Pharmacol., 2014, $5,77$.

126 E. Rideau, R. Dimova, P. Schwille, F. R. Wurm and K. Landfester, Chem. Soc. Rev., 2018, 47, 8572-8610.

127 E. B. Zhulina and O. V. Borisov, Macromolecules, 2012, 45, 4429-4440.

128 S. Yorulmaz Avsar, M. Kyropoulou, S. Di Leone, C.-A. Schoenenberger, W. P. Meier and C. G. Palivan, Front. Chem., 2019, 6, 645.

129 J. S. Lee and J. Feijen, J. Controlled Release, 2012, 161, 473-483.

130 C. LoPresti, H. Lomas, M. Massignani, T. Smart and G. Battaglia, J. Mater. Chem., 2009, 19, 3576-3590.

131 M.-C. Jones and J.-C. Leroux, Eur. J. Pharm. Biopharm., 1999, 48, 101-111.

132 K. Kita-Tokarczyk, J. Grumelard, T. Haefele and W. Meier, Polymer, 2005, 46, 3540-3563.

133 Q. Chen, H. Zhao, T. Ming, J. Wang and C. Wu, J. Am. Chem. Soc., 2009, 131, 16650-16651.

134 L. Caire da Silva, S. Cao and K. Landfester, ACS Macro Lett., 2021, 10, 401-405.

135 A. Harada and K. Kataoka, Science, 1999, 283, 65.

136 H. Cabral, K. Miyata, K. Osada and K. Kataoka, Chem. Rev., 2018, 118, 6844-6892.

137 P. L. Chariou, O. A. Ortega-Rivera and N. F. Steinmetz, ACS Nano, 2020, 14, 2678-2701.

138 S. Iqbal, M. Blenner, A. Alexander-Bryant and J. Larsen, Biomacromolecules, 2020, 21, 1327-1350.

139 S. Rameez, H. Alosta and A. F. Palmer, Bioconjugate Chem., 2008, 19, 1025-1032.

140 A. Kishimura, A. Koide, K. Osada, Y. Yamasaki and K. Kataoka, Angew. Chem., Int. Ed., 2007, 46, 6085-6088.

141 Y. Jiang, J. Zhang, F. Meng and Z. Zhong, ACS Nano, 2018, 12, 11070-11079.
142 T. Einfalt, R. Goers, I. A. Dinu, A. Najer, M. Spulber, O. Onaca-Fischer and C. G. Palivan, Nano Lett., 2015, 15, 7596-7603.

143 L. Messager, J. R. Burns, J. Kim, D. Cecchin, J. Hindley, A. L. B. Pyne, J. Gaitzsch, G. Battaglia and S. Howorka, Angew. Chem., Int. Ed., 2016, 55, 11106-11109.

144 M. Lomora, F. Itel, I. A. Dinu and C. G. Palivan, Phys. Chem. Chem. Phys., 2015, 17, 15538-15546.

145 M. Kumar, M. Grzelakowski, J. Zilles, M. Clark and W. Meier, Proc. Natl. Acad. Sci. U. S. A., 2007, 104, 20719.

146 J. R. Burns, E. Stulz and S. Howorka, Nano Lett., 2013, 13, 2351-2356.

147 I. Neamtu, A. G. Rusu, A. Diaconu, L. E. Nita and A. P. Chiriac, Drug Delivery, 2017, 24, 539-557.

148 E. Mauri, G. Perale and F. Rossi, ACS Appl. Mater. Interfaces, 2018, 1, 6525-6541.

149 H.-Q. Wu and C.-C. Wang, Langmuir, 2016, 32, 6211-6225. 150 K. Landfester, Angew. Chem., Int. Ed., 2009, 48, 4488-4507. 151 L.-P. Lv, Y. Zhao, N. Vilbrandt, M. Gallei, A. Vimalanandan, M. Rohwerder, K. Landfester and D. Crespy, J. Am. Chem. Soc., 2013, 135, 14198-14205.

152 K. Maruya-Li, C. Shetty, A. M. Jazani, N. Arezi and J. K. Oh, ACS Omega, 2020, 5, 3734-3742.

153 K. Kolouchova, O. Sedlacek, D. Jirak, D. Babuka, J. Blahut, J. Kotek, M. Vit, J. Trousil, R. Konefał, O. Janouskova, B. Podhorska, M. Slouf and M. Hruby, Biomacromolecules, 2018, 19, 3515-3524.

154 D.-H. N. Tran, T. H. Nguyen, T. N. N. Vo, L. P. T. Pham, D. M. H. Vo, C. K. Nguyen, L. G. Bach and D. H. Nguyen, J. Appl. Polym. Sci., 2019, 136, 47544.

155 M. Elsabahy and K. L. Wooley, Chem. Soc. Rev., 2012, 41, 2545-2561.

156 K. Landfester, in Colloid Chemistry II, ed. M. Antonietti, Springer, Berlin, 2003, pp. 75-123.

157 A. E. Ekkelenkamp, M. R. Elzes, J. F. J. Engbersen and J. M. J. Paulusse, J. Mater. Chem. B, 2018, 6, 210-235.

158 M. S. Alkanawati, F. R. Wurm, H. Thérien-Aubin and K. Landfester, Macromol. Mater. Eng., 2018, 303, 1700505.

159 K. Piradashvili, E. M. Alexandrino, F. R. Wurm and K. Landfester, Chem. Rev., 2015, 116, 2141-2169.

160 K. Landfester and C. K. Weiss, Adv. Polym. Sci., 2010, 229, 1-49.

161 S.-M. Jo, S. Jiang, R. Graf, F. R. Wurm and K. Landfester, Nanoscale, 2020, 12, 24266-24272.

162 R. Thiramanas, M. Li, S. Jiang, K. Landfester and V. Mailänder, Cells, 2020, 9, 2043.

163 S.-M. Jo, F. R. Wurm and K. Landfester, Nano Lett., 2020, 20, 526-533.

164 S.-M. Jo, F. R. Wurm and K. Landfester, ACS Appl. Mater. Interfaces, 2018, 10, 34230-34237.

165 Y. Zhao, L.-P. Lv, S. Jiang, K. Landfester and D. Crespy, Polym. Chem., 2015, 6, 4197-4205.

166 O. S. Abu Abed, C. Chaw, L. Williams and A. A. Elkordy, Sci. Rep., 2018, 8, 13158.

167 A. V. Kabanov and S. V. Vinogradov, Angew. Chem., Int. Ed., 2009, 48, 5418-5429. 
168 Q. Zhu, X. Chen, X. Xu, Y. Zhang, C. Zhang and R. Mo, Adv. Funct. Mater., 2018, 28, 1707371.

169 J. K. Oh, R. Drumright, D. J. Siegwart and K. Matyjaszewski, Prog. Polym. Sci., 2008, 33, 448-477.

170 M. Machtakova, S. Wirsching, S. Gehring, K. Landfester and H. Thérien-Aubin, J. Mater. Chem. B, 2021, 9, 8389-8398.

171 M. Fichter, K. Piradashvili, A. Pietrzak-Nguyen, L. Pretsch, G. Kuhn, S. Strand, M. Knuf, F. Zepp, F. R. Wurm, V. Mailänder, K. Landfester and S. Gehring, Biomaterials, 2016, 108, 1-12.

172 D. Chenthamara, S. Subramaniam, S. G. Ramakrishnan, S. Krishnaswamy, M. M. Essa, F.-H. Lin and M. W. Qoronfleh, Biomater. Res., 2019, 23, 20.

173 J. W. Hickey, J. L. Santos, J.-M. Williford and H.-Q. Mao, J. Controlled Release, 2015, 219, 536-547.

174 S. Chen, Y. Zhong, W. Fan, J. Xiang, G. Wang, Q. Zhou, J. Wang, Y. Geng, R. Sun, Z. Zhang, Y. Piao, J. Wang, J. Zhuo, H. Cong, H. Jiang, J. Ling, Z. Li, D. Yang, X. Yao, X. Xu, Z. Zhou, J. Tang and Y. Shen, Nat. Biomed. Eng., 2021, 5, 1019-1037.

175 D. V. Haute and J. M. Berlin, Ther. Delivery, 2017, 8, 763-774.

176 S. M. Moghimi and H. M. Patel, Adv. Drug Delivery Rev., 1998, 32, 45-60.

177 X. Duan and Y. Li, Small, 2013, 9, 1521-1532.

178 X. Xie, Q. Tao, Y. Zou, F. Zhang, M. Guo, Y. Wang, H. Wang, Q. Zhou and S. Yu, J. Agric. Food Chem., 2011, 59, 9280-9289.

179 B. Wang, T. Yuan, L. Zha, Y. Liu, W. Chen, C. Zhang, Y. Bao and Q. Dong, Mol. Pharmaceutics, 2021, 18, 1470-1479.

180 S. M. S. Shahriar, J. M. An, M. N. Hasan, S. S. Surwase, Y.-C. Kim, D. Y. Lee, S. Cho and Y.-K. Lee, Nano Lett., 2021, 21, 4666-4675.

181 J. C. Sung, B. L. Pulliam and D. A. Edwards, Trends Biotechnol., 2007, 25, 563-570.

182 C. Xu, H. Tian, P. Wang, Y. Wang and X. Chen, Biomater. Sci., 2016, 4, 1646-1654.

183 Q. Zhang, Z. Shen and T. Nagai, Int. J. Pharm., 2001, 218, 75-80.

184 M. M. A. Abdel-Mottaleb, D. Neumann and A. Lamprecht, Eur. J. Pharm. Biopharm., 2011, 79, 36-42.

185 B. Iqbal, J. Ali and S. Baboota, Int. J. Dermatol., 2018, 57, 646-660.

186 R. Alvarez-Román, A. Naik, Y. N. Kalia, R. H. Guy and H. Fessi, J. Controlled Release, 2004, 99, 53-62.

187 L. Busch, Y. Avlasevich, P. Zwicker, G. Thiede, K. Landfester, C. M. Keck, M. C. Meinke, M. E. Darvin, A. Kramer, G. Müll5er, M. Kerscher, J. Lademann and A. Patzelt, Int. J. Pharm., 2021, 597, 120339.

188 M. E. Davis, Z. Chen and D. M. Shin, Nat. Rev. Drug Discovery, 2008, 7, 771-782.

189 T. Lima, K. Bernfur, M. Vilanova and T. Cedervall, Sci. Rep., 2020, 10, 1129.
190 A. Zielińska, F. Carreiró, A. M. Oliveira, A. Neves, B. Pires, D. N. Venkatesh, A. Durazzo, M. Lucarini, P. Eder, A. M. Silva, A. Santini and E. B. Souto, Molecules, 2020, 25, 3731.

191 M. Faria, M. Björnmalm, K. J. Thurecht, S. J. Kent, R. G. Parton, M. Kavallaris, A. P. R. Johnston, J. J. Gooding, S. R. Corrie, B. J. Boyd, P. Thordarson, A. K. Whittaker, M. M. Stevens, C. A. Prestidge, C. J. H. Porter, W. J. Parak, T. P. Davis, E. J. Crampin and F. Caruso, Nat. Nanotechnol., 2018, 13, 777-785.

192 S. Jesus, M. Schmutz, C. Som, G. Borchard, P. Wick and O. Borges, Front. Bioeng. Biotechnol., 2019, 7, 261.

193 A. Kermanizadeh, N. R. Jacobsen, M. Roursgaard, S. Loft and P. Møller, Heliyon, 2017, 3, e00458.

194 Y. Liu, M. Kong, C. Feng, K. K. Yang, Y. Li, J. Su, X. J. Cheng, H. J. Park and X. G. Chen, Colloids Surf., B, 2013, 103, 345-353.

195 S. K. Jena and A. T. Sangamwar, Carbohydr. Polym., 2016, 151, 1162-1174.

196 K. Sonaje, Y.-H. Lin, J.-H. Juang, S.-P. Wey, C.-T. Chen and H.-W. Sung, Biomaterials, 2009, 30, 2329-2339.

197 S. E.-S. Radwan, M. S. Sokar, D. A. Abdelmonsif and A. H. El-Kamel, Int. J. Pharm., 2017, 526, 366-379.

198 M. Sharma, S. Sharma, V. Sharma, K. Sharma, S. K. Yadav, P. Dwivedi, S. Agrawal, S. K. Paliwal, A. K. Dwivedi, J. P. Maikhuri, G. Gupta, P. R. Mishra and A. K. S. Rawat, Int. J. Biol. Macromol., 2017, 104, 1345-1358.

199 K. Tahara, T. Sakai, H. Yamamoto, H. Takeuchi, N. Hirashima and Y. Kawashima, Int. J. Pharm., 2009, 382, 198-204.

200 M. Kolter, M. Ott, C. Hauer, I. Reimold and G. Fricker, J. Controlled Release, 2015, 197, 165-179.

201 G. Romero, I. Estrela-Lopis, J. Zhou, E. Rojas, A. Franco, C. S. Espinel, A. G. Fernández, C. Gao, E. Donath and S. E. Moya, Biomacromolecules, 2010, 11, 2993-2999.

202 S. Uchida and K. Kataoka, J. Biomed. Mater. Res., Part A, 2019, 107, 978-990.

203 S. M. Moghimi, P. Symonds, J. C. Murray, A. C. Hunter, G. Debska and A. Szewczyk, Mol. Ther., 2005, 11, 990-995.

204 M. A. Gosselin, W. Guo and R. J. Lee, Bioconjugate Chem., 2001, 12, 989-994.

205 V. Knorr, V. Russ, L. Allmendinger, M. Ogris and E. Wagner, Bioconjugate Chem., 2008, 19, 1625-1634.

206 S. Sanati, S. Taghavi, K. Abnous, S. M. Taghdisi, M. Babaei, M. Ramezani and M. Alibolandi, Gene Ther., 2021, DOI: 10.1038/s41434-021-00234-0.

207 D. Liu, F. Yang, F. Xiong and N. Gu, Theranostics, 2016, 6, 1306-1323.

208 K. Smietana, M. Siatkowski and M. Møller, Nat. Rev. Drug Discovery, 2016, 15, 379-380.

209 K. W. Hunter, Nat. Rev. Cancer, 2012, 12, 144-149. 